\title{
Recent advances in metamaterial split-ring-resonator circuits as biosensors and therapeutic agents
}

\author{
Sohini RoyChoudhury ${ }^{1, a}$, Vaishali Rawat ${ }^{2, a}$, Ahmed Hasnain Jalal ${ }^{1}$, S. N. Kale ${ }^{2}$ and Shekhar Bhansali ${ }^{1, *}$ \\ ${ }^{1}$ BioMEMS and Microsystems Laboratory, Department of Electrical \& Computer Engineering, Florida International \\ University, Miami, Florida 33174, United States
}

${ }^{2}$ Department of Applied Physics, Defense Institute of Advanced Technology, Deemed University, Girinagar, Pune-411025, India

${ }^{\mathrm{a} B o t h}$ authors contributed equally

KEYWORDS: metamaterial; microwave; terahertz; plasmon; electromagnetic; dielectric; refractive index; resonance

\begin{abstract}
Potential applications of thin film metamaterials are diverse and their realization to offer miniaturized waveguides, antennas and shielding patterns are on anvil. These artificially engineered structures can produce astonishing electromagnetic responses because of their constituents being engineered at much smaller dimensions than the wavelength of the incident electromagnetic wave, hence behaving as artificial materials. Such micro-nano dimensions of thin film metamaterial structures can be customized for various applications due to their exclusive responses to not only electromagnetic, but also to acoustic and thermal waves that surpass the natural materials' properties. In this paper, the recent major advancements in the emerging fields of diagnostics (sensors) and therapeutics involving thin film metamaterials have been reviewed and underlined; discussing their edge over conventional counterpart techniques; concentrating on their design considerations and feasible ways of achieving them. Challenges faced in sensitivity, precision, accuracy and factors that interfere with the degree of performance of the sensors are also dealt with, herein.
\end{abstract}

\section{1) Introduction}

Sensors are of vital importance to several applications, especially for their development for chemical and biosensing purposes. Electrical investigation of biological materials has been performed over the past century, using both conventional electrodes and microelectrodes. Owing to well-established micromachining techniques, various kinds of biosensing mechanisms have been developed based on the principle of surface plasmon resonance (SPR)(Dostálek et al., 2005)'(Oh et al., 2004), fluorescence(Pickup et al., 2005)'(Viveros et al., 2006), electromechanical transduction(Fritz et al., 2000)'(Lee et al., 2004), nano-materials(Guan et al., 2005)'(Alivisatos, 2004) and other electrochemical(Murphy, 2006)'(Wang, 2006) or other physical processes, such as quartz crystal microbalance (QCM)(Janshoff et al., 2000), cantilevers etc.(Daniels and Pourmand, 2007)'(Ziegler, 2004). These biosensors, though being precise and accurate in detection, face significant limitations as some require laboratory based methods which make these systems more complex with highly sophisticated equipments involving multistep extraction/purification of samples(Singh et al., 2014) with intensive sample preparation and off-site postverification, features which make these techniques both cumbersome and expensive. Biosensors in general have been developed on two basic aspects: one is based on an extremely accurate and precise detection principle like an SPR and a micro-cantilever biosensor; whereas the other is based on developing a low-cost, on-site sensing device such as disposable biosensors(Abayomi et al., 2006)'(Muhammad-Tahir and Alocilja, 2004). Among these, the latter concept of biosensors has received more attention because of its simple and easy detection ability despite being less accurate along with lesser sensitivity compared to other sensing techniques. Micro-calorimeter falls under the latter type of biosensors which has a simple and easy detection scheme(Winter and Höhne, 2003)'(Sun et al., 2011) however, with a drawback of complex fabrication. Conventional sensors also pose issues of selectivity; which happens to be an equal challenge for both types of sensors. With traditional sensing procedures showing such limitations, efforts are on to explore some un-conventional methods with the utilization of some alternative possible sensing techniques like those which are metamaterial based; because of the extraordinary properties shown by these thin film metamaterial configurations. This has been an offshoot of the recently explored unconventional sensing procedures using nano-structured materials, which are being explored due to their ability to "see", "manipulate and "arrange" micromolecules on a "self-assembled" route, creating a path for making nano-structured artificial patterns a reality in the submicron regime. Based on the fundamental idea that a compact form factor can offer considerable enhancement in performance (Bhansali and Vasudev, 2012), metamaterials are artificially engineered electromagnetic materials that are assemblies of multiple individual elements, consisting of periodically arranged metallic elements much less than a wavelength of incident electromagnetic (EM) wave, in size(Chen et al., 2012). The primary research in metamaterials investigates negative index metamaterials which can influence electromagnetic wave beams in interesting ways and display striking electromagnetic properties like backward propagation, reverse Doppler effect, reverse Vavilov- 
Cerenkov effect(Chen et al., 2012)'(Veselago, 1968), negative refraction (Chen et al., 2012)'(Shelby et al., 2001), diffraction-limit breaking imaging(Chen et al., 2012)'(Pendry, 2000), cloaking(Chen et al., 2012)'(Pendry et al., 2006), and so on. These properties strongly depend on the geometry of metamaterial molecules rather than their composition(Chen et al., 2012)'(Pendry, 2006). Apart from negative refraction, structural arrangements achieving negative permittivity and permeability in a desired frequency, which is not exhibited by any natural material, are also considered as metamaterials. Hence, metamaterials exhibit extremely unique sets of properties which find potential applications in imaging (superlenses)(Chen et al., 2012)'(Pendry, 2000)'(Fang et al., 2005), slow light(Chen et al., 2012)'(Bai et al., 2010), data storage(Chen et al., 2012)'(Wuttig and Yamada, 2007), optical switching(Chen et al., 2012)'(Kind et al., 2002), miniaturized antennae/devices(Dong and Itoh, 2012; Itoh, 2012) and so on. Metamaterials based sensing devices in recent times have been widely researched for numerous sensing applications, with both resonant and non-resonant Composite Right Left Handed (CRLH based) metamaterial types been envisaged as potential candidates for sensing purpose in areas like displacement(Horestani et al., 2013)'(Naqui et al., 2011), rotation(Horestani et al., 2013), dielectric characterization(Withayachumnankul et al., 2013)'(Rawat et al., 2014) including strain sensing(Melik et al., 2010)'(Li et al., 2013), mass flow sensor, differential sensor, temperature sensor, level sensor(Schueler et al., 2012)'(Schussler et al., 2011). These materials are apt for sensing applications as they offer high miniaturization and large values of Qfactor which makes them highly sensitive to environmental changes. Such properties of metamaterials also suggest their suitability for material sensing which include solid dielectrics(Boybay and Ramahi, 2012), liquids(Ebrahimi et al., 2014), hybrid fuels, hazardous chemicals'(Rawat et al., 2014)'(Rawat et al., 2015)'(Rawat et al., 2016), gas sensing(Zarifi et al., 2016)(Kaushik et al., 2015)and biomolecules(Lee et al., 2012)'(Clark et al., 2009). Metamaterial-based sensors in general have given a new headway towards novel systems for sensing. In this paper, the design and working of recent metamaterial-based sensors typically for biosensing and therapeutic applications have been reviewed, thus showing their principles of operation and detection based on the sensitivity of the various existing thin film metamaterial based sensors, how they differ from and show superior sensitivity and performance from conventional sensors which gives them huge future prospects along with challenges, these sensors face in their development and mechanism. The mathematical and theoretical treatment of such sensors is discussed first, which includes the electromagnetic theoretical base and simulation treatments to the structure. The later part deals with explicit examples of the ways in which such structures are used (or can be used) for sensing and therapeutics.

\section{2) Physics of metamaterials}

When a wave is incident on a material, the change in its electromagnetic response is governed by the two fundamental parameters i.e. permittivity $(\varepsilon)$ and permeability $(\mu)$. These material properties are, in general, unique for every material owing to their distinctive atomic configurations. Metamaterials are artificially designed structures with sizes smaller than the wavelength of the incident wave $(\lambda / 10$ in general); which can manipulate the wave to achieve the desired response. Since, the structures are much smaller than the wavelength; they are not resolved by the incident e-m wave and seem invisible to the wave. Therefore, all the structures collectively behave as new material/medium with properties very distinct and exclusive from its constituent materials where each one of them individually behave as electric dipoles just like atoms in case of natural materials. Almost all naturally existing materials have a positive $\varepsilon$ and $\mu$ throughout the electromagnetic spectrum expect for metals (negative $\varepsilon$ epsilon near visible frequencies) and ferrites (negative $\mu$ mu at lower frequencies). However, there is no existing material found till date to have both negative $\varepsilon$ and $\mu$ over the same frequency range. The reason being, such materials if in existence will be highly dispersive and dissipative in nature(Ramakrishna, 2005). Further, $\varepsilon$ and $\mu$ are related to refractive index (n) by the following equation

$$
n=\sqrt{\varepsilon \mu} \ldots \ldots . . . .(1)
$$

From the equation above, it can be inferred that materials which have both positive $\varepsilon$ and $\mu$ have real and positive refractive index and allow wave propagation. Materials with either one of the parameters being negative will inhibit wave propagation as refractive index becomes imaginary. This is the reason that metals are excellent reflectors at optical wavelengths. Materials with both negative $\varepsilon$ and $\mu$ have a real but negative valued refractive index (to prevent the violation of work-energy causality) and also allow wave propagation. This striking phenomenon of achieving negative refractive index is the most interesting property of metamaterials. In this case, the direction of propagation is reversed as group and phase velocity become anti-parallel(Smith and Kroll, 2000).

The plot below (Figure 1) shows the classification of metamaterials on the basis of $\varepsilon$ and $\mu$. Most of the dielectric materials lie in the first quadrant with $\varepsilon$ and $\mu$ as positive. These are called doubly positive media (DPS). When only electrical permittivity becomes negative, it is an electrical metamaterial or $\varepsilon$-negative media $(\mathrm{ENG})$, these lie in the second quadrant; while materials with negative magnetic permeability fall in the fourth quadrant and are known as magnetic metamaterial or $\mu$-negative media (MNG). Upon both parameters becoming negative, the materials fall in the third quadrant and are known negative index metamaterials or doubly negative media (DNG)(Metamaterials: Physics and Engineering Explorations, 2006).

\section{3) Sensing mechanism of metamaterials}

The basic principle operation of metamaterial sensing is based on the change in transmission/reflection coefficients (S-parameters) in terms of frequency and/or Q-factor when there is a change in the sensed parameters such as mass loading, dielectric change and mechanical force, due to variation in $\mu / \varepsilon$ or refractive index of the metamaterial resonator(Chen et al., 2012). Another method for sensing is based on the phase of the reflection and transmission coefficients where the shift in the phase of the transmission and reflection coefficients is analyzed. In the phase detection method, the resolution of the system is further increased (Boybay and Ramahi, 2012). The operational frequency in 
this case must be in the region where the phase has a very high slope to obtain higher sensitivity. Microwave metamaterial sensors are in general fabricated using the UV-photolithography technology, which eases the design, mitigates loss, and simplifies measurements as well as data acquisition. The connection to the readout circuitry can be easily realized in this case, with transmission lines coupled to the resonators.

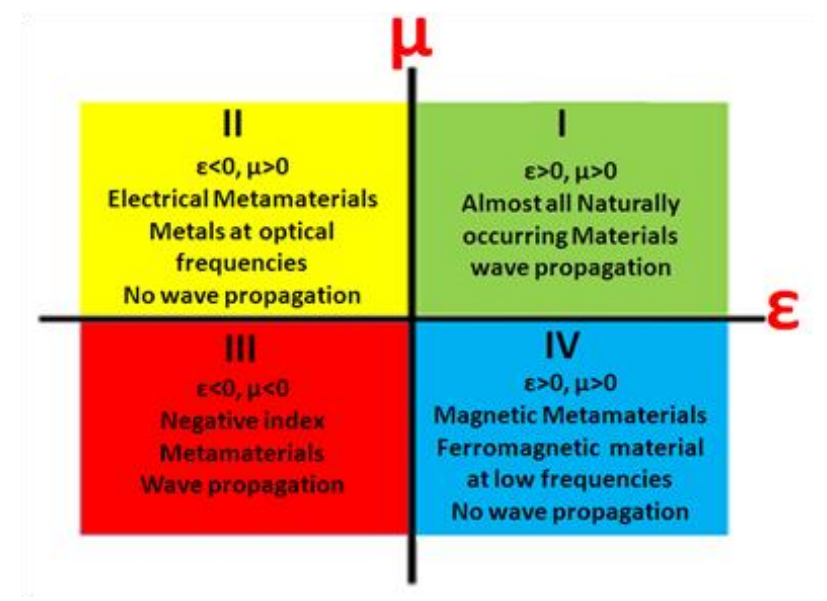

Figure 1. Graph of $\varepsilon$ vs. $\mu$, showing various quadrants and the materials' behaviour in each of these divisions the graph of behaviour in each of these divisions

Thermally tunable enhanced transmission of microwaves was studied through a sub-wavelength aperture by a dielectric metamaterial resonator providing a boost for several microwave applications. With a transmission coefficient of $\sim 20 \mathrm{~dB}$, this dielectric metamaterial resonator was seen to be stable over a temperature range from $20^{\circ} \mathrm{C}$ to $9^{\circ} \mathrm{C}$ with adjustable transmission peak from $11.6 \mathrm{GHz}$ to $12.1 \mathrm{GHz}(\mathrm{Guo}$ et al., 2016). Since metamaterial sensors are based on structural response i.e. sub-wavelength in nature, the response exhibited is, in general, high resonance over narrow frequency range, thus exhibiting a high Q-factor. High Q-factor of the sensor is advantageous as it offers increased sensitivity and resolution, which are significant parameters for sensing. Further, design of simple structures is sufficient for sensor operation. The sensor is also remarkably miniaturized in size owing to its sub-wavelength resonance which is one of the reasons for improved sensitivity as high resonance is achieved over a small region(Yang, 2011). The interaction between electromagnetic waves and materials has therefore been proven favorable for the analysis of the structure, composition, and function of biological components, such as biological cells and organic tissues, and for the monitoring of industrial processes (Schueler, 2012). In this sense, metamaterials are most suitable for sensing applications throughout the electromagnetic spectrum.

\section{4) Common metamaterial configurations}

Among metamaterial structures, Split Ring Resonator (SRR) was one of the first conceived and till date most widely researched designs. SRR exhibits negative permeability when the magnetic field is incident perpendicular to the plane of the SRR. The underlying concept of SRR resonance exhibiting negative permeability is briefly explained ahead, for general understanding.

Consider a metal ring placed in an oscillating electromagnetic wave with the magnetic field polarized perpendicular to the ring surface. The magnetic field induces a circular current in the ring according to Faraday's Law, which produces a magnetic flux (purely inductive and non-resonant) opposing the external magnetic field. Further, capacitance is introduced by providing a gap in the metallic ring to introduce a resonance behavior. Due to the split in the rings, a circulating current results in a build-up of charge across the gap, stored as capacitance. Capacitance is more effectively introduced when two rings are placed concentrically with their gaps opposite each other (known as double SRRs) as shown in Figure 2(a). Double SRRs provide an edge from single SRR by lowering the resonance frequency and minimizing the electric polarizability(Cai and Shalaev, 2009). These are extensively studied and explored among SRR-based metamaterial structures. SRR can also be modeled into an LC equivalent circuit since it behaves as a quasi-static LC circuit in response to the external field. The equivalent circuit of a unit cell SRR is shown in Figure 2(b). The resonance frequency, $\omega_{0}$ of the SRR is given by the following equation(Padilla et al., 2006);

$$
\omega_{0}=2 \pi f_{0}=(L C)^{-1 / 2}
$$

where, $\mathrm{L}$ and Care equivalent inductance and capacitance decided by the dimensions of the SRR. For frequencies below $\omega_{0}$, currents in the SRR can keep up with the driving force produced by the externally varying magnetic field and a positive response is achieved. However, as the frequency of the external magnetic field is increased, the currents can no longer keep up and eventually begin to lag, resulting in an outof-phase or negative response. Generally, frequency dependent permeability is given by the following equation;

$$
\mu(\omega)=1-\frac{\omega_{p}^{2}}{\omega^{2}-\omega_{0}^{2}+i \gamma \omega} \ldots \ldots \ldots
$$


where, $\omega_{\mathrm{p}}$ is the plasma frequency and $\gamma$ is the damping coefficient. In case of SRR, these parameters are dependent on the structure dimensions. Therefore, the permeability for double circular SRR is derived as below(Liu and Zhang, 2011);

$$
\mu(\omega)=1-\frac{F \omega^{2}}{\omega^{2}-\omega_{0}^{2}+\mathrm{i} \gamma \omega} \ldots \ldots \ldots
$$

where,

$$
\begin{aligned}
F & =\frac{\pi r^{2}}{a^{2}} \ldots \ldots . .(5) \\
\omega_{0} & =\sqrt{\frac{3 d c^{2}}{\pi^{2} r^{3}} \ldots \ldots \ldots(6)} \\
\gamma & =\frac{2}{r \sigma \mu_{0}} \ldots \ldots . .(7)
\end{aligned}
$$

where, $a$ is lattice constant, $r$ is average radius of the external SRR, $d$ is the distance between the two SRR, $c$ is the ring thickness and $\sigma$ is the conductivity of metal rings.

Another interesting structure which has been vastly researched for sensing applications is complementary split ring resonator (CSRR). CSRR is considered as dual SRR based on Babinet's principle(F Falcone et al., 2004). In this case, two concentric rings are removed from a conductive surface to achieve a CSRR, as shown in Figure 2(c). Neglecting metal thickness and all losses, a perfectly dual behavior is expected for the CSRR. SRR is mainly considered as a resonant magnetic dipole that is excited by an axial magnetic field whereas CSRR essentially behaves as an electric dipole (with the same frequency of resonance) excited by an axial electric field. In this circuit, the inductance and capacitance switch places in an SRR circuit i.e. the inductance of the SRR model is substituted by the capacitance and viceversa(F. Falcone et al., 2004)'(Baena et al., 2005). CSRR structures have also been extensively used for sensing applications mostly in the microwave range(Boybay and Ramahi, 2012)'(Ebrahimi et al., 2014)'(Rawat et al., 2015). These electrical metamaterial (double CSRR) structures for hybrid fuel and high energy materials have been explored, due to their ease of sensing mechanism and high sensitivity (Rawat et al., 2014)'(Rawat et al., 2015)'(Rawat et al., 2016). Apart from these, a lot of variants of SRRs have been designed and fabricated such as spiral resonators, broadside coupled split-ring resonator (BC-SRR), two-layer multi spiral resonator (TL-MSR), the broad-side coupled spiral resonator with four turns, the open split-ring resonator (OSRR), and the open complementary split-ring resonator (OCSRR)(DuranSindreu et al., 2011). Symmetrical-Ring Structure, Omega Structure, coupled "S" shaped structure are also a few metamaterial structures further developed and researched after split ring resonators. Further, transmission line (TL) configurations such as CRLH (composite rightleft-handed) transmission line are also available for sensing applications in the microwave range. The CRLH line is a transmission line approach to obtain non-resonant, low loss and broadband behavior. The TL structures are constituted of series inter-digital capacitors $\mathrm{C}_{\mathrm{L}}$ and shunt inductors $\mathrm{L}_{\mathrm{L}}$, in the microstrip line intended to provide left-handedness as shown in Figure 2(d). Figure 2(e) depicts the equivalent circuit of CRLH transmission line. The coupling leads to a substantial change in electromagnetic properties significant for the sensor application and different from SRR based configurations. SRR arrays have a distinct resonance frequency that generally exhibit small band behavior, whereas in a CRLH TL, resonance is expanded to various resonances allowing broadband operation(Caloz and Itoh, 2005)'(Schueler et al., 2012).

\section{5) Metamaterials for biosensing and therapeutic applications}

Metamaterials can exhibit certain extraordinary electromagnetic properties as described above. Hence, they have been attracting great interest among both scientists and engineers alike as they facilitate label-free and cost effective bio-analyte detection(Chen et al., 2012). The mechanism of metamaterial based biosensors also mainly depends on the change in the resonant frequency due to the binding of different bio-molecules onto metamaterial resonators. To achieve a higher sensitivity, the sensor needs a high Q-factor in its frequency response which results in high concentration of electric/magnetic field in a smaller region and enables the detection of minute changes in the desired ambience- Using metals will show an improvement in film quality with time through lateral grain growth, similar to what has been observed at room temperature in continuous metal films' deposition. The sensing structures can act as dielectric substrates, providing both support and a material to sample and embed the analytes, while the patterning metamaterial on the substrate can offer a platform where the resonance shifts, mainly because of alterations in the SRR capacitance induced by the added analytes. This can be utilized for quantitative biochemical sensing applications(Chen et al., 2012). 


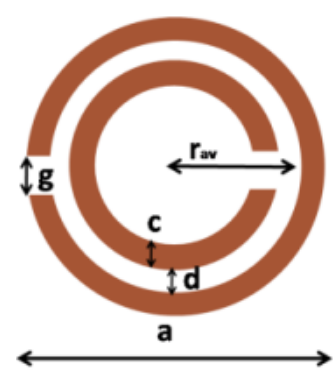

(a)

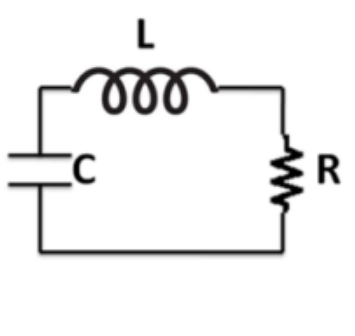

(b)

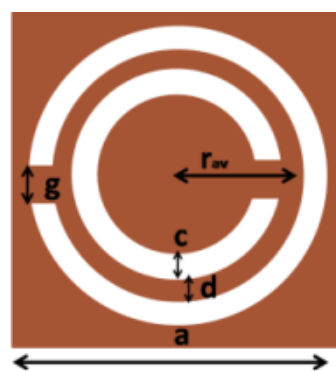

(c)

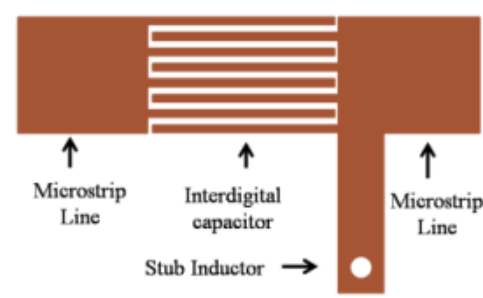

(d)

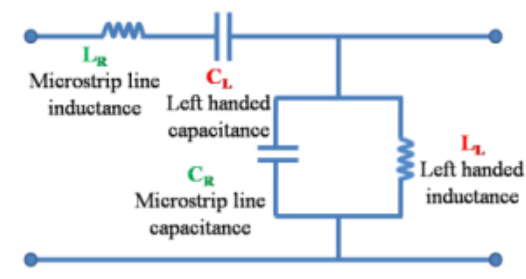

(e)

Figure 2. (a) Schematic of a double-turn split-ring resonator with its dimension (b) equivalent RLC tank circuit of a single split ring resonator, valid when it is electrically small (c) Schematic of a double complementary split-ring resonator and (d) Layout of the unit cell of the microstrip Left Handed-Transmission Line prototype, including a series interdigital capacitor and a shunt shorted stub inductor for left handedness (e) Equivalent circuit of Composite Right Left Handed(CRLH) metamaterial transmission line

\section{a) Biochemical and bio-molecule detection}

In the past few years, resonator based metamaterials have been extensively research for biochemical and bio-molecule detection for example DNA, proteins, hemoglobin etc. Split-ring resonator (SRR) structures which can be designed to be very compact having dual band characteristics; is a metamaterial offering negative permeability in a time-varying H-field component of perpendicularly polarized wave incident on its surface(Pendry et al., 1999)'(Smith et al., 2004)'(Liu et al., 2007). Lee et al. experimentally demonstrated a biosensing device based on split ring resonator array, which can be compared to an LC resonator to operate at microwave frequencies.

DNA hybridization has been recognized with a shift in the resonant frequency, when thiol-linked single stranded-DNA is immobilized onto a gold surface and is coupled with the complementary-DNA. It was thus confirmed that in the microwave regime, the metamaterial resonator can be utilized as a DNA sensing element. In their recent efforts, they have also proposed(Lee et al., 2012) an asymmetric splitring resonator(for higher sensitivity), as a biosensing transducer for detection of highly sensitive and label-free stress biomarkers using two biomarkers, cortisol and a-amylase with a resonance frequency at $13.06 \mathrm{GHz}$. These stress biomarkers are closely related to changes in human emotion and mood disorders such as trauma and mental depression. Therefore, quantification of the concentration of a-amylase as well as cortisol in saliva is an essential part of psychotherapy. The reaction between cortisol-bovine serum albumin and free cortisol is employed for cortisol detection, while alpha-amylase is directly detected by its antigen-antibody reaction. After patterning and gold plating the SRR, a masking layer (-10-20 $\mu$ m-thickness) made of solder mask PCB ink was used for confinement of bio-molecular binding to the surface of the target resonator, excluding the part of the resonator that was the active sensing region and the edge contact pads used for measurement) as shown in Figure 3(a)(Lee et al., 2012). The limit of detection and sensitivity of the proposed sensing device was achieved about $1 \mathrm{ng} \mathrm{ml}^{-1}$ and $1.155 \mathrm{MHz} \mathrm{ng}^{1} \mathrm{ml}^{-1}$, respectively. Using Ricin Chain-A as a mode analyte, fabrication of a thermally stable electrochemical immuno-sensor has also been reported by Bhansali et. al, with single-domain antibodies (sdAb) employed as recognition elements for direct detection at temperatures greater than $4^{0} \mathrm{C}$ at the nanoscale. Such electrochemical sensors can well be integrated as part of a portable device for point of care immuno-sensing purposes, with a maximum stability up to $40^{\circ} \mathrm{C}$ and a shelf life of about 7 days(Singh et al., 2016). 1-D and 2-D ZnO nanostructures were also studied for selective detection of cortisol using a simple one step sonochemical approach through a label free, highly sensitive and selective electrochemical immuno-sensor which can be integrated with micro-fluidics and a miniaturized potentiostat for point-of-care personalized health monitoring. These nanostructures reportedly exhibit improved chemical stability, higher catalytic performance and biocompatibility for integration on a wearable platform, with a detection limit of $1 \mathrm{pM}$, which is reportedly 100 times better than conventional enzyme linked immunosorbant immunoassay (ELISA)(Vabbina et al., 2015).In addition to this, K. Jaruwongrungsee et.al successfully developed and demonstrated a microstrip-coupled SRR integrated with a polydimethyl-siloxane (PDMS) chamber for real-time, label-free and quantitative detection of IgG. The SRR is patterned on a general 
microwave printed circuit board which resonates at $2 \mathrm{GHz}$ while the microfluidic chamber is fabricated by casting of PDMS. SRR was immobilized with Anti- Immunoglobulin G (IgG) for IgG detection by a standard covalent immobilization using Cystamine. The PDMS chamber was aligned and clamped on the circuit board and the electromagnetic response of the SRR sensor was continuously monitored when IgG analytes were flowed through the chamber. The sensor exhibited frequency-shift response in the concentration range of 50-200 $\mu \mathrm{g} / \mathrm{ml}$ (Jaruwongrungsee et al., 2015).

Further, Humeyra Caglayan et. al(Caglayan et al., 2010) reported that SRR structures can also be used for bioassay applications for improving the assay time and sensitivity. Figure 3(b) depicts the single square SRR structure fabricated on general cu-clad substrate with a resonance frequency around $2.45 \mathrm{GHz}$ with a transmission of $-20 \mathrm{~dB}$. The transmission properties of a single SRR structure are measured by using a vector network analyzer and two monopole antennas as receiver and transmitter antennas. A microcuvette of $10 \mu \mathrm{L}$ volume capacity is drilled at the gap of the SRR. The basic principle of bioassay technique is based on the focusing of microwaves on the microcuvette for uniform heating and creating a thermal gradient between the assay medium and the silver colloids for the rapid completion of the bioassay steps. The demonstration of the ultrafast bioassays was accomplished by using a model biotin-avidin bioassay. Alongside, an identical ELISA was also carried out on commercially available HTS wells at room temperature incubation instead of microwave heating. While the room temperature bioassay without microwave heating took 70 min to complete, the identical bioassay took less than 2 min to complete by using SRR structures with microwave heating. A lower detection limit of $0.01 \mathrm{nM}$ for biotinylated-bovine serum albumin 100-fold lower than the room temperature bioassay was observed. H. Torun et. al have designed an antenna-coupled split-ring resonator-based microwave sensor, for biosensing applications as shown in Figure 3(c)(Torun et al., 2014). The sensor comprises single circular split ring resonator and integrated monopole antennae on top of a dielectric substrate. With ground plane at the backside of the substrate, integrated antennae are used to excite the device and the resonant frequency of the device was measured at $2.12 \mathrm{GHz}$. The schematic of the experimental setup is shown in Figure 3(d). The device is demonstrated as a resonant bio-molecular sensor where the interactions between heparin and fibroblast growth factor 2 are probed. The surface of parylene was incubated with FGF-2 molecules with a concentration of $140 \mu \mathrm{g} \mathrm{ml}^{-1}$. A $10 \mu \mathrm{l}$ droplet was placed on the sensor and molecules were allowed to anchor on the surface of the device. When the surface was dried, a $20 \mu 1$ droplet of heparin with a concentration of $10 \mu \mathrm{g} \mathrm{ml}^{-1}$ was placed immediately afterwards. The sensitivity of the device was obtained as $3.7 \mathrm{MHz}\left(\mu \mathrm{g}^{-1} \mathrm{ml}^{-1}\right)$ with respect to changes in concentration of heparin.

Another antenna-based sensor with novel combinational design of metamaterial i.e. composite right/left-handed (CRLH) based transmission line operating at $1.69 \mathrm{GHz}$ was proposed by Fu-Chieh Chang for the applications of biosensors(Chang et al., 2012). The antenna biosensor's left-handed $(\mathrm{LH})$ capacitance $\left(\mathrm{C}_{\mathrm{L}}\right)$ is attributed to the inter-digital capacitors' coupling between the patch and the antenna biosensor's LH inductance $\left(\mathrm{L}_{\mathrm{L}}\right)$ is due to the shorted-stub inductances to ground. The right-handed $(\mathrm{RH})$ effects are due to the capacitive $\left(C_{R}\right)$ coupling between the patch and ground plane, and the current flow atop the patch $\left(L_{R}\right)$. The resonant frequency shifts from $1.69 \mathrm{GHz}$ to $1.63 \mathrm{GHz}$ under different concentrations of water-ethanol solutions (3\% to $100 \%$ ) with equivalent permittivity of 79 to 14 . To further improve the sensitivity of the metamaterial biosensor, a tip-shaped SRR metamaterial thin-film sensor with an improved Q-factor was studied by Xun-jun He and Yue Wang in 2010(He et al., 2010). Rectangular asymmetric double split resonators with a similar miniaturized design can also be used for thin-film sensing techniques with a structure consisting of two-dimensional, periodic resonator arrays, where a single unit cell placed inside a rectangular waveguide is sufficient for sensing (Al-Naib et al., 2008). This design concentrates the field components into a small area thereby increasing volumetric sensitivity of the device. Further, a novel metamaterialbased microwave thin-film sensor incorporating multiple split ring resonators in a compact nested structure was proposed in 2011 by He $e t$ al. as illustrated in Figure 3(e)(He et al., 2011). In this nested structure with more split gaps, the sensing performance was investigated using three-dimensional full-wave electromagnetic solver simulations. Its results showed low non-linearity error and high sensitivity at lower frequency of operation with extended miniaturized size and design flexibility for biosensing. In addition to this, a very recent theoretical study was carried out by Markus Wellenzohna and Martin Brandl on a biosensing device based on microwave split-ring resonators (SRRs), developed and optimized for operation in the ultra-high frequency regime, by means of extensive finite element method (FEM) simulations(Wellenzohn and Brandl, 2015). Their simulation results indicate a frequency shift of about $100.9 \mathrm{MHz}$ for a hydrogel based bio-functionalization with a layer thickness of $3 \mu \mathrm{m}$.

Metamaterials for biosensors have also been extensively researched in higher frequency regimes i.e. from terahertz to optical wavelengths. This frequency region of the electromagnetic spectrum provides great promise in the field of biosensing because of low photon energy and some specific features like THz time-domain spectroscopy to distinguish between drugs, detect skin cancer, and characterize DNA, proteins and/or carbohydrates. Improving the sensitivity of metamaterials by the optimization of the structure parameters as proposed by Debus et. al, is a well known approach. His design posed asymmetric split ring resonators with sharp resonances as non-specific biosensors with a frequency shift (Debus and Bolivar, 2007). Wu et. al studied a label-free and specific biosensor for streptavidin - agarose (SA), fabricated based on $\mathrm{THz}$ metamaterial, functionalized by octadecan thiols and biotins for which both low as well as high frequency resonant modes (LFRM \& HFRM) from metamaterials were found applicable for the detection of SA(Wu et al., 2013). SRR based planar terahertz metamaterials fabricated on thin film substrates show increased sensitivity and improved performance as compared to bulk and such materials have been realized to show the ability of sensing a dielectric layer of $200 \mathrm{~nm}$ thickness, the sensing modality of which changes based on the capacitance of the SRR as shown by O'Hara et al.(Tao et al., 2010)'(O'Hara et al., 2008). Waveguide sensors have also proven useful for sensing thin films of water by increasing the effective interaction length(Zhang and Grischkowsky, 2004). Moreover, $\mathrm{THz}$ filters and micro-resonators were explored for sensing certain analytes by the shift in frequency stimulated by them on the resonant 
reaction of the device. This was reported to have improved sensitivity to the binding state of DNA samples by approximately 103 times over conventional free-space time-domain spectroscopy(Nagel et al., 2002)'(Brucherseifer et al., 2000).
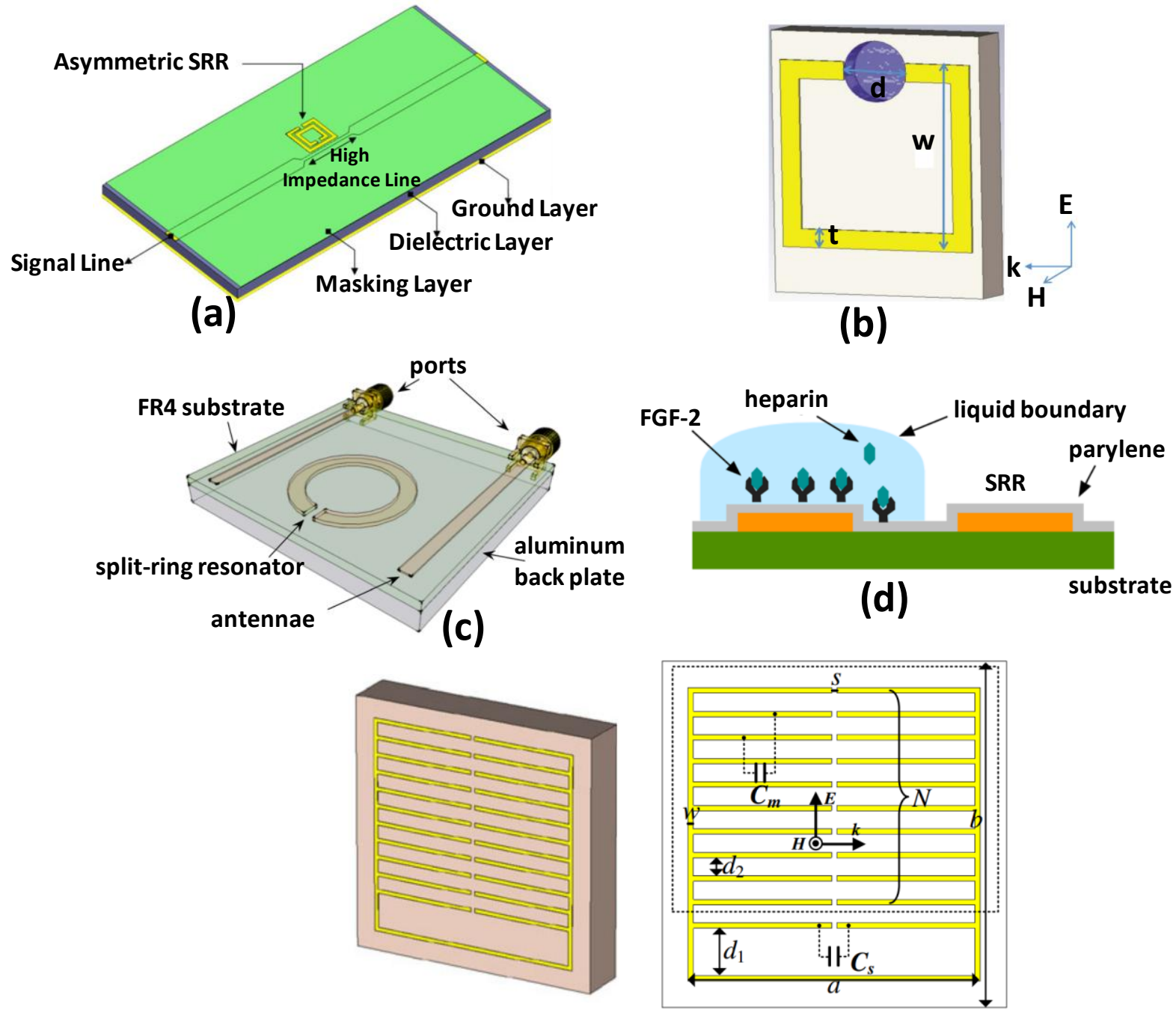

(e)

Figure 3. (a) Schematic of a SRR based on a high impedance microstrip line for label free detection (b) Schematic of the SRR structure printed on a circuit board for bioassay application. (c) Biosensor structure and integrated monopole antennas on a dielectric substrate (d)

experimental setup presenting the functionalization of the device with bio-molecules (e) Nested Split Ring Resonator structure

Ranjan Singh et. al reported an approximately $85 \%$ modulation of the basic inductive-capacitive resonance with an experimental observation of three distinct resonances (LC, dipole and quadrupole) in split ring resonators (SRRs) for horizontal and vertical electric field polarizations at normal incidence with $\mathrm{THz}$ time domain spectroscopy; showing that increased asymmetry leads to the growth of high quality factor electric quadrupole resonance which can be investigated further for bio-sensing applications in the THz region (Singh et al., 2010). As seen in the Figure 4(a) below, the first one has the orientation of the incident terahertz field perpendicular to the SRR gap inducing weak LC resonance, while in the latter figure the field is along the SRR gap in order to excite the regular LC and the dipole mode resonances(Singh et al., 2010). The current distribution in these structures in the different resonant modes is shown in Figure 4(b)(Singh et al., 2010). Introducing asymmetry leads to this kind of current distribution that scatters the electromagnetic field very weakly and dramatically reduces the coupling to the free space causing a reduction in the losses which can lead to sharp resonance. This quadrupole resonance is inaccessible in the symmetrical structure(Singh et al., 2010).

High Q factor of electric quadrupole resonances has been explored for sensing and narrow band filtering purposes by Yoshida et al. with metallic meshes working as band-pass filters in the THz region as shown in Figure 4(c[i])(Yoshida et al., 2009), with a high-efficiency transmission peak in the wavelength region greater than the grating period as shown in Figure 4(c[ii])(Yoshida et al., 2009). This high- 
efficiency transmittance was investigated using finite-difference time-domain (FDTD) electromagnetic simulations for high-sensitivity observations by focusing on abrupt change in transmittance that appeared when the THz wave was obliquely incident onto the mesh.

Christian et. al by numerical simulation and selective loading; explored an approach to detect small amounts of chemical and biochemical probe material with a frequency selective surface made from asymmetric split ring resonators when excited with free space radiation with the use of a concentrated E-field. Due to interference, resonance occurs with a change in the frequency response (Debus and Bolivar, 2007). Ogawa et. al demonstrated highly sensitive detection of small amounts of protein horseradish peroxidase i.e. $500 \mathrm{pg} \mathrm{mm}^{-2}\left(11 \mathrm{fmol}^{2}\right.$ of horseradish peroxidase printed on the metallic mesh(Yoshida et al., 2007) through distinct shifts in transmission resonance frequency using a similar label-free biosensor. Lately, Sijiang Wang et.al proposed a polarization-insensitive metamaterial biosensor composed of metallic SRRs suitable to work together with the linear polarized femto-second laser pumped terahertz time-domain spectroscopy. The metamaterial structure is made of aluminum on a PET substrate with a thickness of $35 \mu \mathrm{m}$. The biosensor structure was made polarization insensitive by providing a symmetric slit on every side of the ring was simulated and analyzed using simulation software COMSOL, thereafter fabricated and investigated experimentally for bovine serum albumin (BSA). The metamaterial biosensor obtains a $275 \mathrm{GHz}$ frequency shift for a $3 \mathrm{mmol}^{-1}$ aqueous solution of BSA, with a resolution of $17.7 \mu \mathrm{mol}^{-1}$ regardless of the orientation of the biosensor (Wang et al., 2016).

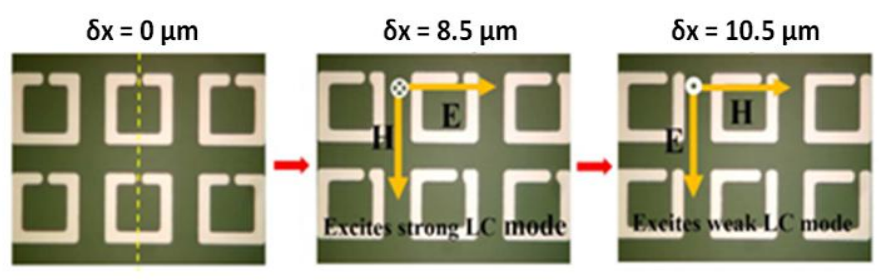

(a)

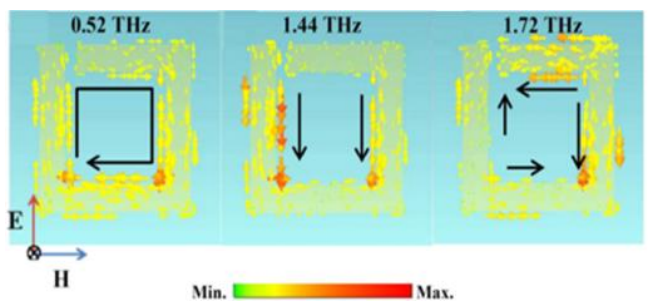

(b)
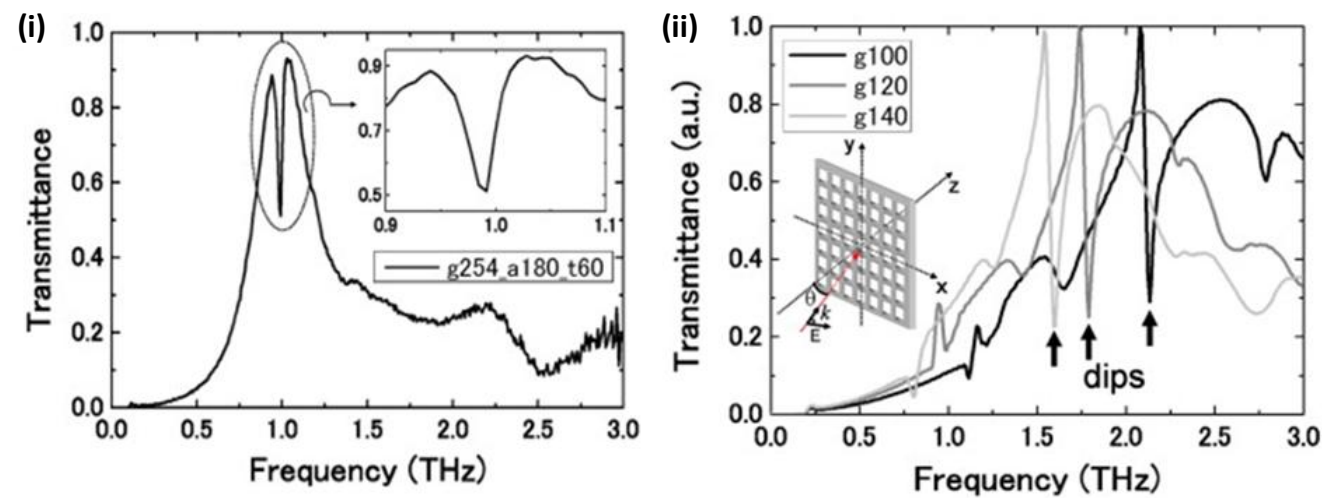

(c)

Figure 4. (a) Microscopic image of SRR arrays with increase in gap in the SRR by different values of ' $\delta$ ' from 0 um to 10.5 um from the center, (b) Surface current distribution for asymmetry parameter $\delta \mathrm{x}=10.5 \mu \mathrm{m}$ for vertical E field polarization at different frequencies of weak LC resonance, dipole resonance and quadrupole resonance, (c) [i] Transmission spectra of metallic mesh [ii] Dip frequency reliance on the opening ratio for the square-lattice metallic mesh

b) Role of plasmons in biosensing

Metamaterials display certain properties which may often appear exotic and intriguing in the optical domain. Although, it is well known that conventional surface plasmon polariton sensors as biosensors already possess many desirable traits making them the devices of choice for many applications, metamaterials ensure new degrees of design freedom and introduce novel operating principles, overcoming the design limitations of conventional plasmon sensors. Label-free plasmonic biosensors rely either on surface plasmon polaritons or on localized surface plasmons on continuous or nanostructured noble-metal surfaces to detect molecular binding events. A. V. Kabashin et. al. demonstrated a plasmonic metamaterial capable of supporting a guided mode in a porous nanorod layer for sensing of standard streptavidin-biotin affinity model, thereby recording considerable improvement in detection limits of small analytes, compared with conventional label-free plasmonic devices (Kabashin et al., 2009). The metamaterial provides enhanced sensitivity to refractive-index variations of the medium between the rods (more than 30,000 $\mathrm{nm}$ per refractive-index unit) due to substantial overlap between the probing 
field and the active biological substance incorporated between the nanorods and a strong plasmon-mediated energy confinement inside the layer.

In case of metamaterial based surface plasmon resonance (SPR) nano biosensor, photonic energy propagates to the surface of the metamaterial layers as packets of electrons called surface plasmons. SPR sensors consist of an ultra thin $(\sim 50 \mathrm{~nm})$ metamaterial layer (usually gold or silver) that is fabricated on dielectric in which photonic energy travels. Supporting this incidence, p-polarized light satisfies the resonance condition where a charge density oscillated at $100 \mathrm{~nm}$ around the surface of the metamaterials, where the resonance wave is expressed as surface plasmon wave (SPW). The parameters that decide the resonant condition are dielectric constants of the metal and medium, wavelength and incident angle. For biosensing applications, the metamaterial layer is incorporated with the 'sensing' dielectric layer that causes shifting of resonance frequency, by changing the angle of orientation of the metal layer or wavelength or reflection intensity in presence of biochemical molecules(Erickson et al., 2008).

There are three modes of detection of SPR sensors that are shown in Figure 5 (Erickson et al., 2008).

i. Angular SPR Nano-biosensor

ii. Spectral SPR Nano-biosensor

iii. Local SPR Nano-biosensor

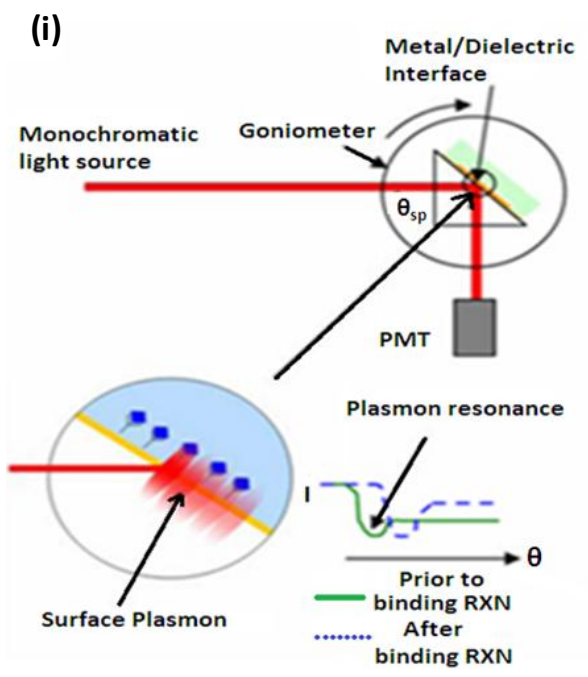

(ii)
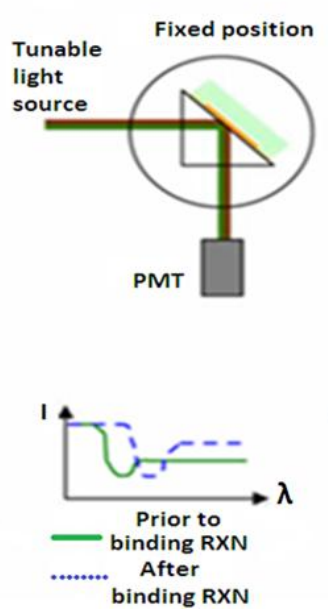

(iii)

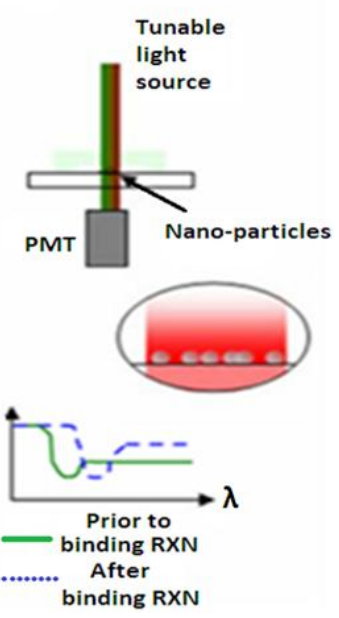

Figure 5. Different SPR sensor systems

Angular SPR Nano-biosensor is the most commonly used sensing methodology of SPR sensing. In this pattern of system, a thin metamaterial array is incorporated with several bio-recognition agents. As a consequence, the incident angle is shifted and an alteration occurs in dielectric constant. The quantification of target biochemical species directly depends on the shifting of the angle of incidence $\left(\boldsymbol{\theta}_{\mathrm{sp}}\right)$ of plasmon excitation.

This angle of excitation is defined as follows:

$$
\theta_{\mathrm{sp}}=\frac{1}{n_{p}} \sqrt{\frac{\varepsilon_{m}(\lambda) \varepsilon_{d}}{\varepsilon_{m}+\varepsilon_{d}}} \ldots \ldots \ldots
$$

where, $\mathrm{n}_{\mathrm{p}}$ is the refractive index of the prism, $\varepsilon_{\mathrm{m}}(\lambda)$ is the dielectric constant of the metal film which varies as a function of the excitation wavelength, $\lambda$, and $e_{d}$ which represents the dielectric constants of the dielectric layer (Kabashin et al., 2009).

The difference between spectral SPR nano biosensor with angular SPR nano biosensor is nothing but having fixed incident angle and the use of a tunable light source instead of a monochromatic light source. The concentration and bio-affinity property of target bio-molecules measures the change in the resonant wavelength. Its outstanding sensitivity and spectral approach instead of measuring incidence angle 
leads to an image based data collection technique. However, use of the tunable light source enhances the overall cost of the system. Furthermore, the portability also becomes an issue. Local SPR sensing technique is more appropriate for nanostructures' or nanoparticles' sensing because of coupling into plasmon mode on the surface of sub-wavelength scale. The resonant frequency strongly depends on the dielectric constant. However, ion beam or electron beam lithography is applied for the fabrication of this nanostructure based sensor. The most attractive advantages of this sensor are its simplicity of arrangement and multiplexing ability. This sensor is reported as a good biomarkers associated with Alzheimer's disease using sera sample(Haes et al., 2005).

Further, works of Alasdair W. Clark et. al, using electron beam lithography manipulated the nanoscale geometry of Ag split-ring resonators, to tune their optical properties so that they exhibit two independent high frequency plasmon resonance modes for SERRS(Clark et al., 2009). This tailored multimodal, polarization dependent activity enables the split rings to act as discriminating sensors, with each resonance tuned for a particular sensing purpose in molecular diagnostics. The structures are used as multi-wavelength, multi-analyte DNA SERRS sensors, with each resonance tuned to both the absorption wavelength of a differently colored Raman reporter molecule and its corresponding laser excitation wavelength. They have shown the ability of each resonance to independently sense small concentrations of a single DNA type from within a mixed population, an effect of the split ring's dichroic response on the SERRS signal and the sensor's limit of detection of each resonance mode. Luigi La Spada et. al fabricated a metamaterial-based sensor, operating in the infrared frequency range for hemoglobin measurements(Spada et. al, 2012). The structure consists of a planar array of complementary square inclusions. The sensor resonant frequencies are designed to coincide with the main absorbance peaks of oxy-hemoglobin and deoxygenated hemoglobin, in order to reveal their presence and concentrations. The structure response is characterized by multiple resonant frequencies, by changing the electromagnetic wave incident angle. Apart from SRR structures, Zhe Liu et. al studies CSRR metamaterials in the visible region and demonstrates a refractive index sensitive in nanoscale CSRRs for dielectric and biosensing application(Liu et. al, 2012). CSRR based metamaterials were fabricated in nanoscale regime with unit length of $100 \mathrm{~nm}$ and slit width of $30 \mathrm{~nm}$ by top-down nanofabrication technology. These visible responses show a good tunability with the structure's geometry, and are well suited for dielectric detection. From the optical transmission responses of the CSRRs, evident anisotropic visible responses in the waveband of 600-900 nm were observed. The visible spectral resonances were regarded as the combination of SPP and waveguide modes (WMs), which can be used for refractive index sensing in the visible region. The results showed that the CSRRs had a refractive index sensitivity of about $210 \mathrm{~nm}$ RIU. For different incident polarization, the resonance peaks shifted from $780 \mathrm{~nm}(830 \mathrm{~nm})$ to $870 \mathrm{~nm}(935 \mathrm{~nm})$ when the CSRRs were coated with BSA (bovine serum albumin). Moreover, Zoran Jakšić discussed several classes of metamaterials for chemical or biological sensors based on "left-handed" metamaterials, artificial single negative materials, the "zero index" structures with a near zero value of the real part of the refractive index along with different types of structures, including split ring resonator and its variants, wires "fishnet" media, arrays of subwavelength apertures in optically opaque conductive sheets displaying extraordinary optical transmission and devices based on ultrathin metal-dielectric multi-layers utilizing freestanding, self-supported nano-membranes(Jakšić, 2010).

The metamaterial structures can also be fabricated on flexible substrates (like those which are polymer based). Using polymer substrates, smooth, patterned metal films $(\mathrm{Au}, \mathrm{Ag}, \mathrm{Cu})$ can be fabricated with conventional deposition techniques at room temperature, without adhesion or seeding layers, facilitating new photonic and plasmonic nanostructures, including transparent electrical contacts, thin film waveguides, metamaterials, biosensors and high-contrast superlenses. Working on these lines, Xinlong Xu et. al, demonstrated a flexible metamaterial (Metaflex)-based photonic device operating in the visible infrared regime for strain, biological and chemical sensing applications(Xu et al., 2011). The metamaterial structure, consisting of split ring resonators (SRRs) of $30 \mathrm{~nm}$ thick Au or Ag, fabricated on poly (ethylene naphthalate) substrates with a least line width of $\sim 30 \mathrm{~nm}$ by electron beam lithography. The absorption resonances tuned from middle IR to visible range. The Ag U-shaped SRR metamaterials exhibit an electric resonance of $\sim 542 \mathrm{~nm}$ and a magnetic resonance of $\sim 756 \mathrm{~nm}$. The electric and magnetic resonance modes show highly sensitive responses to out-of-plane bending strain, surrounding dielectric media, and surface chemical environment. Due to the electric and magnetic field coupling, the magnetic response gives sensitivity as high as $436 \mathrm{~nm} / \mathrm{RIU}$. The devices show superior responses with a shift of magnetic resonance of $4.5 \mathrm{~nm}$ for nonspecific bovine serum albumin protein binding and $65 \mathrm{~nm}$ for a self-assembled monolayer of 2-naphthalenethiol, respectively. Also recently, Sang-Gil et al.(Sang-Gil Park and Ki-Hun Jeong, 2014) showed another very novel high performance label-free biosensor by using an all dielectric metamaterial in order to avoid metal loss in the high frequency regime. These dielectric metamaterials consist of nanohole arrays in rectangular lattice on a thin silicon substrate. The nanohole arrays were fabricated by using e-beam lithography and silicon reactive ion etching. The biosensor shows high sensitivity and high figure-of-merit of 21 offering new opportunities for biosensing with high performance and reusability.

\section{c) Cancer detection}

The detection of abnormalities in cancerous cells is another important field of research which has seen considerable progress in the past years, though current diagnostics still have many disadvantages. Current methods for detection and diagnosis start with imaging tests such as computerized tomography (CT) scans, X-Rays and magnetic resonance imaging (MRI). After an abnormality is detected, additional tests are made usually with needle biopsies that can be quite invasive and have their own risks. False positives occur often because the test can mistake scar tissues or a benign lump for cancer. Since it has been proven that organic tissues present a clearly identified dielectric behavior which is frequency dependent(Gabriel et al., 1996)'(Dean et al., 2006), methods based on microwaves, where the sensing is made non-invasively; are a very interesting field of study; and combining them with the multiple advantages of metamaterial structures, can lead to a very cost-effective method of cancer detection (Boybay and Ramahi, 2007)'(Schaefer et al., 2004). On these lines, a prototype sensor 
based on microstrip line-excited SRR array has been explored by Margarita Puentes et. al (Puentes et al., 2011). Within the array it is possible to track each resonant peak individually and from its frequency shift, relative change in the dielectric properties of the tissue can be identified. A schematic of the sensor array for analyzing these organic tissues, is shown in Figure 6(a) (Puentes et al., 2011).

With this information, in-homogeneities can be located; since the position where the alteration took place can also be extracted. Another study done by Luigi La Spada et.al, proposes a low-cost, compact, and high performing metamaterial-based biosensor operating in the GHz regime(La Spada et al., 2011). The sensor is designed to detect the presence of a tumor by means of permittivity and absorption measurements. In addition to this, Dagmar Faktorová et. al presented a novel waveguide sensor for non-destructive determination of biological tissues and dielectric properties measurement in the microwave frequency band(Faktorova et al., 2012). This approach investigates the dielectric properties of biological material with the implementation of artificial metamaterial structures with negative permittivity and permeability over the open waveguide sensor, to increase the sensing properties of a classical waveguide sensor and achieve the optimal design of metamaterial structures, for waveguide sensors operating in microwave X-band. The microwave open waveguide sensors tuned with metamaterial structures works in reflection - transmission mode; and can be used to investigate dielectric properties of biological tissues changing at their pathological processes.

\section{d) Bio-imaging and hyperthermia}

The exotic property of negative refractive index exhibited by metamaterials has been widely researched for superlenses which can also contribute to medical imaging techniques. Though, theoretical concept of negative lens was given by Pendry in 2000, the first superlenses at microwave frequencies were demonstrated in 2004, with a negative refractive index. This provided a three times better resolution than the regular diffraction limits and in 2005; N. Fang et al. illustrated the first near field superlens which exceeded the diffraction limit(Fang et al., 2005).
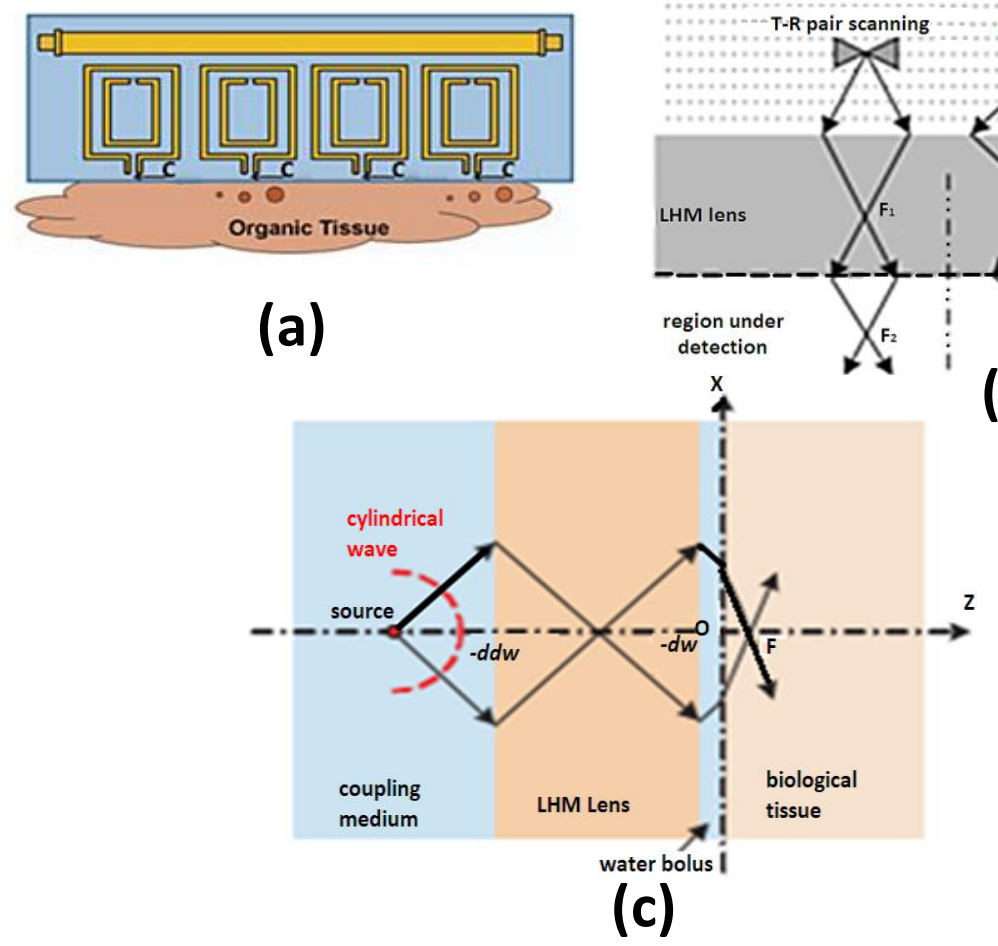

Figure 6. (a) SRR sensor array for analysis of organic tissues (b) Design for target detection and imaging with flat LHM lens at microwave frequencies (c) Hyperthermia scheme with flat LHM lens

Usage of flat Left Handed Metamaterial (LHM) lens with superior resolution has the latent of achieving not only finer imaging resolution but also simpler in-depth scanning, making a less complicated system design for near-field microwave target detection and imaging execution, which is shown in Figure 6(b) (Wang et al., 2007). Therapeutics is another arena where metamaterials lens have been proven beneficial and shown significant improvement in results compared to conventional counterparts. Therapeutics is basically concerned with the treatment of disease and the action of remedial agents. Hyperthermia is one such domain where a diseased tissue is exposed to elevated temperatures for a period of time, has long been regarded as a promising treatment for cancer and other diseases. Further, metamaterial superlenses have also shown useful for treatment of hyperthermia(Tao and Wang, 2012). In hyperthermia, microwaves are transmitted into a patient for annihilation of the ailing tissues by heating them to a temperature which can cause their death. This treatment entails directing 
a restricted dose of heat to the affected tumor. The negative-refractive index (NRI) of the flat LHM lens which focuses the electromagnetic field of the generator can produce an appropriate focusing spot in the biological tissue as needed, in microwave superficial tumor hyperthermia treatment shown in Figure 6(c) below(Gong and Wang, 2009). Such a microwave focusing device consisting of a planar array of split-ring resonators placed between two parallel metallic plates and fed by an antenna exciting the split-rings, was also studied for this treatment. The device was modeled as a homogeneous uni-axial slab of negative permeability placed between two metallic plates. Both the fields and the temperature distribution in a model of breast tissue and a tumor were examined(Velazquez-Ahumada et al., 2011). Further, David Vrba et. al investigated the heating performance of novel multi-element array hyperthermia applicators based on a metamaterial based zero-eth order mode antenna. The antenna is suitable for assembly into large array applicators and has shown to provide more homogenous heating $\left(41-45^{\circ} \mathrm{C}\right)$ of large target areas than possible with typical waveguide antennas. These applicators can heat effectively down to 3-4 cm depth using a water bolus temperature of $30^{\circ} \mathrm{C}$ with moderate or high level blood perfusion. The data demonstrated that these metamaterial antenna array applicators can uniformly heat large areas of tissue up to $560 \mathrm{~cm}^{2}$ (Vrba et al., 2016).

\section{e) Sub-terahertz frequency wireless communication}

With increased demand for enhanced data rates in wireless communication, metamaterials help to bridge the THz gap with electrical control, miniaturized size, and readily integrable fabrication processes of structurally reconfigurable metamaterials, making them a supreme candidate for the realization of various terahertz communication devices like electrically controllable terahertz delay lines, buffers, and tunable data-rate channels (Pitchappa et al., 2016). Planar metamaterials reportedly being able to achieve electromagnetically induced transparency (EIT), structurally reconfigurable metamaterials for active switching of near-field coupling in conductively coupled, orthogonally twisted split ring resonators (SRRs) operating in the terahertz spectral region have been reported, with the individual SRRs designed with similar inductive-capacitive resonant frequencies (Pitchappa et al., 2016).These reconfigurable conductively coupled structures designed with curved cantilevers integrated into the SRR for tuning the dark SRR resonance as seen in Fig 7(a), shift the resonant frequency with complete modulation of EIT leaving the conductive coupling between the SRRs unaltered. Active control of this phenomenon, realized by near field coupling, through excitation of sharp radiative mode resonance in the dark resonator amidst a broader absorption spectra of the bright resonator, this has been seen as in Fig 7(b), to enable tunable active switching group-delay of $\sim 2.5$ ps with EIT modulation contrast of $\sim 50 \%$ (Pitchappa et al., 2016). Inter-coupling distance between the resonators, bright mode and dark mode resonance frequencies can be changed to attain the active control of EIT bandwidth, intensity, and frequency in near field coupled materials.

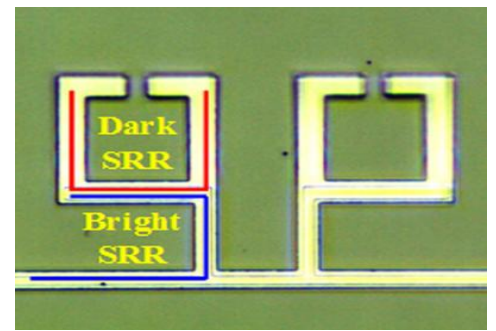

(a)

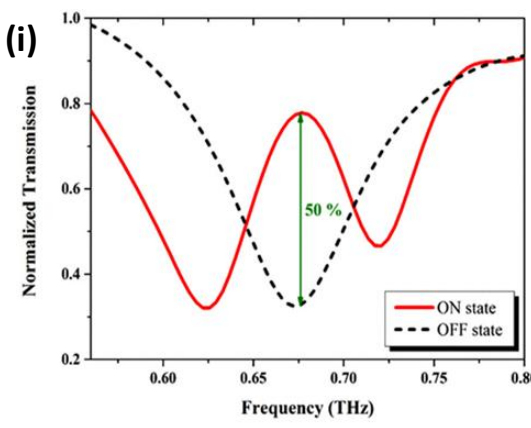

(ii)
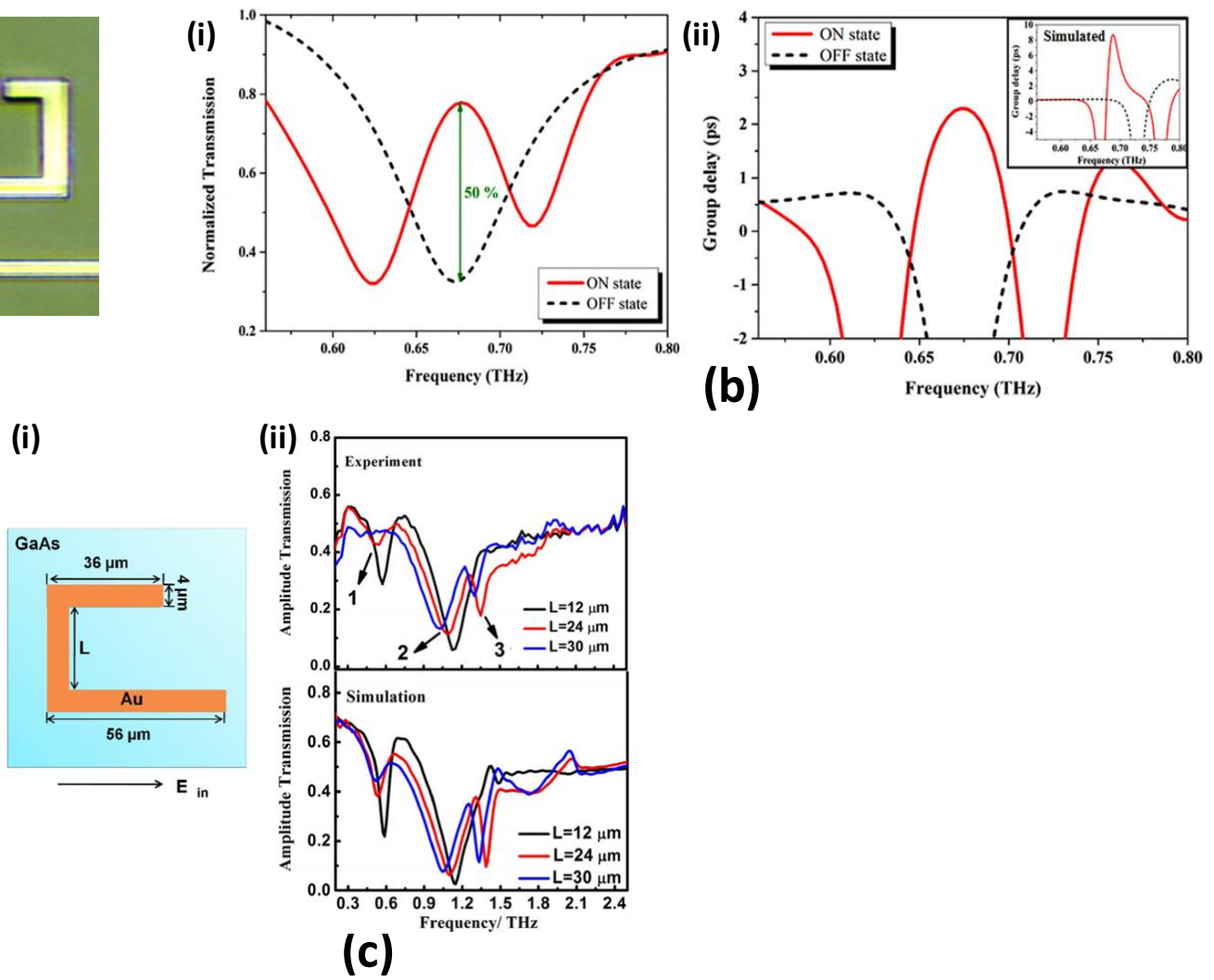

Fig 7: (a) Optical microscopic image of conductively coupled MEMS device with curved cantilevers (b) Measured (i) THz response (ii) Group delay of conductively coupled MEMS device (c) (i) Schematic of asymmetric design structure with perpendicular incident polarization (ii) Obtained amplitude transmission spectra 
Different asymmetric metamaterial design configurations based on the fundamental inductive-capacitive resonance in terahertz metamaterials, modulated by Yang et. al, of double-split ring basic SRR unit have been reported(Yang et al., 2011). Mutlu et. al and Cheng et. al have discussed asymmetric U-shaped SRR metamaterial structures and their ultrathin dual-mode filtering characteristics in the terahertz range with electrically unconnected and connected U-shaped resonator array designs (Mutlu et al., 2011)(Cheng et al., 2015). Based on these, this U-shaped array structure was seen to show intriguing absorption behavior characteristics in the transmission spectra with horizontal and vertical polarizations in response to incident terahertz frequencies. Although with the polarization direction of the incident electric field being parallel to the bottom bars of the asymmetric structure, two absorption dips are seen corresponding to the low frequency LC resonance and high frequency dipole resonance respectively; three absorption dips have been seen with the polarization direction of incidence being perpendicular to the design structure, as depicted in Fig 7(c)(Yang et al., 2016). Devices incorporating terahertz metamaterial attract attention for its great prospects in $\mathrm{THz}$ imaging, filtering and modulation in various biological sensing applications(Yang et al., 2016) with terahertz electromagnetic waves having huge potential to open up unique applications in medical diagnosis and bio sensing coupled with high-speed wireless-communication(Asai and Kawabata, 2016).

\section{6) Conclusion: challenges and future prospects}

Biomedical technologies greatly demand the attention of multidisciplinary efforts with the chemistry of complex oxides, physio-chemistry of different methods for their preparation and investigation, mechanics and strength of such systems and finally medicine as a major stage in biomedicine which is now focusing on the novel techniques based on the functionalities of thin films. Fields of nano-medicine and biosensing have witnessed great progress in the field of metamaterials, further advancing towards a huge range of biomedical applications. Recent advances in interdisciplinary research and molecular diagnostics have led to the rapid development of different classes of biosensors for a wide range of bio-analyte detection with improved sensing characteristics. The interest in applying micro-electromechanical systems (MEMS) technology for biological applications have grown rapidly because of some of its unique features including better performance, high sensitivity, fast responsiveness, temporal control, size, cost and comparable feature sizes (Bhansali and Vasudev, 2012). The attainment of success in the clinical field needs concurrent accomplishment of a stable tissue-implant interface and an appropriate match based on their behavior, of the correlation between the implant and tissue to be replaced(Nanostructured Materials and Coatings for Biomedical and Sensor Applications, 2012). The advent of such devices in medical applications was in the early 1970s with silicon-micromachined disposable blood pressure sensors(Rebello, 2004); and over the last couple of decades there has been an exponential growth in such devices for biomedicine primarily because of device miniaturization, improved functionality, efficient transduction processes, higher reaction rate, lower reagent consumption, lower weight, lower unit cost per device and higher reliability (Bhansali and Vasudev, 2012). Not only do these have the potential of improving functionality of existing surgical devices, but they also provide improved surgical outcomes, lower risks and help control costs by providing real time feedback on the operations undertaken(Rebello, 2004). Metamaterial-based sensors in general have given a new head-way towards novel systems for sensing, pushing the sensitivity of biosensors towards singlemolecule detection limit, high throughput sensor arrays, bringing in new modalities to bio-imaging and new investigative techniques capable of detecting single bio-molecules, their analysis and manipulation; and to revolutionize both the diagnosis as well as the treatment of several ailments. As discussed in the above paragraphs, it can be concluded that metamaterial biosensors have numerous advantages such as high sensitivity, resolution, miniaturization, cost effectiveness and label free detection. However, like any emerging field, it also faces many challenges; some of which include optimization of the adsorption and desorption (AD) rate of analyte particles on the metamaterial device to achieve desired stable results(Djurić et al., 2002) and replacing metal structures with dielectric configuration to avoid losses occurring at metal-dielectric interface at higher frequency regime, using high-k low loss dielectric substrates for fabricating extremely miniaturized and stable structures. Also, incorporating flexible structures will permit the sensor to realize flexible installments inside any device. The development of nanoscale lithographic methods on polymer-based substrates is a key requirement to improve the spatial resolution and performance of flexible devices. This will enable the development and integration of an unprecedented structure for a label-free detection system that conventional biosensors cannot achieve. Designing a compact, rugged and handy device for on-site detection and deployment in harsh environments is a requisite. Numerous in-lab techniques already exist, which are well developed and are extremely sensitive; whereas on-site detection based sensors are still a potential challenge and a major area of research. Despite the challenges, thin film metamaterial biosensors have a very optimistic future due to various advantages they possess. Metamaterials based biosensors are highly miniaturized label free and reversible with high sensitivity and selectivity which make them highly favorable from conventional sensors; and they can be envisaged as potential devices for future scenarios. The field is increasingly attracting more research and with the advent of micro- and nano-fabrication techniques, numerous innovative sensing methodologies can further be realized using metamaterials.

\section{Author information}

\section{Corresponding Author}

*Email: sbhansa@fiu.edu, Phone: +13053484439

\section{Acknowledgements}

This work was supported by the National Science Foundation (NSF) ASSIST Nanosystems ERC under Award Number (EEC-116048) 


\section{References}

Abayomi, L.A., Terry, L.A., White, S.F., Warner, P.J., 2006. Development of a disposable pyruvate biosensor to determine pungency in onions (Allium cepa L.). Biosens. Bioelectron. 21, 2176-9. doi:10.1016/j.bios.2005.10.024

Alivisatos, P., 2004. The use of nanocrystals in biological detection. Nat. Biotechnol. 22, 47-52. doi:10.1038/nbt927

Al-Naib, I.A.I., Jansen, C., Koch, M., 2008. Thin-film sensing with planar asymmetric metamaterial resonators. Appl. Phys. Lett. 93, 083507. doi:10.1063/1.2976636

Asai, H., Kawabata, S., 2016. Emission of Circularly Polarized Terahertz Wave From Inhomogeneous Intrinsic Josephson Junctions. IEEE Trans. Appl. Supercond. 26, 1-4. doi:10.1109/TASC.2016.2515853

Baena, J.D., Bonache, J., Martin, F., Sillero, R.M., Falcone, F., Lopetegi, T., Laso, M.A.G., Garcia-Garcia, J., Gil, I., Portillo, M.F., Sorolla, M., 2005. Equivalent-circuit models for split-ring resonators and complementary split-ring resonators coupled to planar transmission lines. IEEE Trans. Microw. Theory Tech. 53, 1451-1461. doi:10.1109/TMTT.2005.845211

Bai, Q., Liu, C., Chen, J., Cheng, C., Kang, M., Wang, H.-T., 2010. Tunable slow light in semiconductor metamaterial in a broad terahertz regime. J. Appl. Phys. 107, 093104. doi:10.1063/1.3357291

Bhansali, S., Vasudev, A., 2012. MEMS for biomedical applications. Woodhead Pub.

Boybay, M.S., Ramahi, O.M., 2012a. Material Characterization Using Complementary Split-Ring Resonators. IEEE Trans. Instrum. Meas. 61, 30393046. doi:10.1109/TIM.2012.2203450

Boybay, M.S., Ramahi, O.M., 2012b. Material Characterization Using Complementary Split-Ring Resonators. IEEE Trans. Instrum. Meas. 61, 30393046. doi:10.1109/TIM.2012.2203450

Boybay, M.S., Ramahi, O.M., 2007. Double negative metamaterials for subsurface detection. Conf. Proc. ... Annu. Int. Conf. IEEE Eng. Med. Biol. Soc. IEEE Eng. Med. Biol. Soc. Annu. Conf. 2007, 3485-8. doi:10.1109/IEMBS.2007.4353081

Brucherseifer, M., Nagel, M., Haring Bolivar, P., Kurz, H., Bosserhoff, A., Büttner, R., 2000. Label-free probing of the binding state of DNA by timedomain terahertz sensing. Appl. Phys. Lett. 77, 4049. doi:10.1063/1.1332415

Caglayan, H., Cakmakyapan, S., Addae, S.A., Pinard, M.A., Caliskan, D., Aslan, K., Ozbay, E., 2010. Ultrafast and sensitive bioassay using split ring resonator structures and microwave heating. Appl. Phys. Lett. 97, 093701. doi:10.1063/1.3484958

Cai, W., Shalaev, V., 2009. Optical Metamaterials: Fundamentals and Applications. Springer Science \& Business Media.

Caloz, C., Itoh, T., 2005. Electromagnetic Metamaterials: Transmission Line Theory and Microwave Applications. John Wiley \& Sons.

Chang, F.-C., Yin, Y.-Z., Lin, C.-W., Shiu, J., 2012. 4.5.3 A Novel design of Antenna for biosensing applications. Proc. IMCS $2012407-410$. doi:10.5162/IMCS2012/4.5.3

Chen, T., Li, S., Sun, H., 2012. Metamaterials application in sensing. Sensors (Basel). 12, 2742-65. doi:10.3390/s120302742

Cheng, Z., Chen, L., Zang, X., Cai, B., Peng, Y., Zhu, Y., 2015. Ultrathin dual-mode filtering characteristics of terahertz metamaterials with electrically unconnected and connected U-shaped resonators array. Opt. Commun. 342, 20-25. doi:10.1016/j.optcom.2014.12.042

Clark, A.W., Glidle, A., Cumming, D.R.S., Cooper, J.M., 2009. Plasmonic split-ring resonators as dichroic nanophotonic DNA biosensors. J. Am. Chem. Soc. 131, 17615-9. doi:10.1021/ja905910q

Daniels, J.S., Pourmand, N., 2007. Label-Free Impedance Biosensors: Opportunities and Challenges. Electroanalysis 19, 1239-1257. doi:10.1002/elan.200603855

Dean, D., Machado-Aranda, D., Ramanathan, T., Molina, I., Sundararajan, R., 2006. Electrical Properties of Biological Tissues - An Impedance Spectroscopy Study, in: 2006 IEEE Conference on Electrical Insulation and Dielectric Phenomena. IEEE, pp. 357-360. doi:10.1109/CEIDP.2006.311943

Debus, C., Bolivar, P.H., 2007. Frequency selective surfaces for high sensitivity terahertz sensing. Appl. Phys. Lett. 91, 184102. doi:10.1063/1.2805016

Djurić, Z., Jakšić, O., Randjelović, D., 2002. Adsorption-desorption noise in micromechanical resonant structures. Sensors Actuators A Phys. 96, 244251. doi:10.1016/S0924-4247(01)00834-2

Dong, Y., Itoh, T., 2012. Promising Future of Metamaterials. IEEE Microw. Mag. 13, 39-56. doi:10.1109/MMM.2011.2181447

Dostálek, J., Vaisocherová, H., Homola, J., 2005. Multichannel surface plasmon resonance biosensor with wavelength division multiplexing. Sensors Actuators B Chem. 108, 758-764. doi:10.1016/j.snb.2004.12.096

Duran-Sindreu, M., Velez, A., Siso, G., Velez, P., Selga, J., Bonache, J., Martin, F., 2011. Recent Advances in Metamaterial Transmission Lines Based on Split Rings. Proc. IEEE 99, 1701-1710. doi:10.1109/JPROC.2011.2114870

Ebrahimi, A., Withayachumnankul, W., Al-Sarawi, S., Abbott, D., 2014. High-Sensitivity Metamaterial-Inspired Sensor for Microfluidic Dielectric Characterization. IEEE Sens. J. 14, 1345-1351. doi:10.1109/JSEN.2013.2295312

Erickson, D., Mandal, S., Yang, A.H.J., Cordovez, B., 2008. Nanobiosensors: optofluidic, electrical and mechanical approaches to biomolecular detection at the nanoscale. Microfluid. Nanofluidics 4, 33-52. doi:10.1007/s10404-007-0198-8

Faktorova, D., Savin, A., Grimberg, R., 2012. Enhancement of waveguide sensor for biological tissues dielectric properties investigation with metamaterials, in: 2012 35th International Conference on Telecommunications and Signal Processing (TSP). IEEE, pp. 544-548. doi:10.1109/TSP.2012.6256354

Falcone, F., Lopetegi, T., Baena, J.D., Marques, R., Martin, F., Sorolla, M., 2004. Effective negative-/spl epsiv/ stopband microstrip lines based on complementary split ring resonators. IEEE Microw. Wirel. Components Lett. 14, 280-282. doi:10.1109/LMWC.2004.828029

Falcone, F., Lopetegi, T., Laso, M.A.G., Baena, J.D., Bonache, J., Beruete, M., Marqués, R., Martín, F., Sorolla, M., 2004. Babinet principle applied to the design of metasurfaces and metamaterials. Phys. Rev. Lett. 93, 197401. doi:10.1103/PhysRevLett.93.197401

Fang, N., Lee, H., Sun, C., Zhang, X., 2005. Sub-diffraction-limited optical imaging with a silver superlens. Science 308, 534-7. doi:10.1126/science.1108759

Fritz, J., Baller, M.K., Lang, H.P., Rothuizen, H., Vettiger, P., Meyer, E., Güntherodt, H., Gerber, C., Gimzewski, J.K., 2000. Translating biomolecular recognition into nanomechanics. Science 288, 316-8.

Gabriel, S., Lau, R.W., Gabriel, C., 1996. The dielectric properties of biological tissues: III. Parametric models for the dielectric spectrum of tissues. Phys. Med. Biol. 41, 2271-2293. doi:10.1088/0031-9155/41/11/003

Gong, Y., Wang, G., 2009. SUPERFICIAL TUMOR HYPERTHERMIA WITH FLAT LEFT-HANDED METAMATERIAL LENS. Prog. Electromagn. Res. 98, 389-405. doi:10.2528/PIER09091401

Guan, W.-J., Li, Y., Chen, Y.-Q., Zhang, X.-B., Hu, G.-Q., 2005. Glucose biosensor based on multi-wall carbon nanotubes and screen printed carbon electrodes. Biosens. Bioelectron. 21, 508-12. doi:10.1016/j.bios.2004.10.030

Guo, Y., Liang, H., Hou, X., Lv, X., Li, L., Li, J., Bi, K., Lei, M., Zhou, J., 2016. Thermally tunable enhanced transmission of microwaves through a subwavelength aperture by a dielectric metamaterial resonator. Appl. Phys. Lett. 108, 051906. doi:10.1063/1.4941406

Haes, A.J., Chang, L., Klein, W.L., Van Duyne, R.P., 2005. Detection of a biomarker for Alzheimer's disease from synthetic and clinical samples using a 
nanoscale optical biosensor. J. Am. Chem. Soc. 127, 2264-71. doi:10.1021/ja044087q

He, X., Qiu, L., Wang, Y., Geng, Z., Wang, J., Gui, T., 2011. A Compact Thin-Film Sensor Based on Nested Split-Ring-Resonator (SRR) Metamaterials for Microwave Applications. J. Infrared, Millimeter, Terahertz Waves 32, 902-913. doi:10.1007/s10762-011-9807-4

He, X., Wang, Y., Wang, J., Gui, T., 2010. Thin-film sensor based tip-shaped split ring resonator metamaterial for microwave application. Microsyst. Technol. 16, 1735-1739. doi:10.1007/s00542-010-1080-2

Horestani, A.K., Abbott, D., Fumeaux, C., 2013a. Rotation Sensor Based on Horn-Shaped Split Ring Resonator. IEEE Sens. J. 13, $3014-3015$. doi:10.1109/JSEN.2013.2264804

Horestani, A.K., Fumeaux, C., Al-Sarawi, S.F., Abbott, D., 2013b. Displacement Sensor Based on Diamond-Shaped Tapered Split Ring Resonator. IEEE Sens. J. 13, 1153-1160. doi:10.1109/JSEN.2012.2231065

Itoh, T., 2012. Metamaterial-Based Antennas. Proc. IEEE 100, 2271-2285. doi:10.1109/JPROC.2012.2187631

Jakšić, Z., 2010. Optical metamaterials as the platform for a novel generation of ultrasensitive chemical or biological sensors. Metamaterials classes, properties and applications. Nova Science Publishers.

Janshoff, A., Galla, H., Steinem, C., 2000. Piezoelectric Mass-Sensing Devices as Biosensors-An Alternative to Optical Biosensors? Angew. Chem. Int. Ed. Engl. 39, 4004-4032. doi:10.1002/1521-3773(20001117)39:22<4004::AID-ANIE4004>3.0.CO;2-2

Jaruwongrungsee, K., Waiwijit, U., Withayachumnankul, W., Maturos, T., Phokaratkul, D., Tuantranont, A., Wisitsoraat, A., 2015. Real-time and labelfree biosensing with microfluidic-based split-ring-resonator sensor, in: 2015 IEEE 15th International Conference on Nanotechnology (IEEENANO). IEEE, pp. 1091-1094. doi:10.1109/NANO.2015.7388812

Kabashin, A. V, Evans, P., Pastkovsky, S., Hendren, W., Wurtz, G.A., Atkinson, R., Pollard, R., Podolskiy, V.A., Zayats, A. V, 2009. Plasmonic nanorod metamaterials for biosensing. Nat. Mater. 8, 867-71. doi:10.1038/nmat2546

Kaushik, A., Kumar, R., Arya, S.K., Nair, M., Malhotra, B.D., Bhansali, S., 2015. Organic-Inorganic Hybrid Nanocomposite-Based Gas Sensors for Environmental Monitoring. Chem. Rev. 115, 4571-4606. doi:10.1021/cr400659h

Kind, H., Yan, H., Messer, B., Law, M., Yang, P., 2002. Nanowire Ultraviolet Photodetectors and Optical Switches. Adv. Mater. 14, $158-160$. doi:10.1002/1521-4095(20020116)14:2<158::AID-ADMA158>3.0.CO;2-W

La Spada, L., Bilotti, F., Vegni, L., 2011. Metamaterial biosensor for cancer detection, in: 2011 IEEE SENSORS Proceedings. IEEE, pp. 627-630. doi:10.1109/ICSENS.2011.6127103

Lee, H.-J., Lee, H.-S., Yoo, K.-H., Yook, J.-G., 2010. DNA sensing using split-ring resonator alone at microwave regime. J. Appl. Phys. $108,014908$. doi:10.1063/1.3459877

Lee, H.-J., Lee, J.-H., Moon, H.-S., Jang, I.-S., Choi, J.-S., Yook, J.-G., Jung, H.-I., 2012. A planar split-ring resonator-based microwave biosensor for label-free detection of biomolecules. Sensors Actuators B Chem. 169, 26-31. doi:10.1016/j.snb.2012.01.044

Lee, H.-J., Yook, J.-G., 2008. Biosensing using split-ring resonators at microwave regime. Appl. Phys. Lett. 92, 254103. doi:10.1063/1.2946656

Lee, J.H., Yoon, K.H., Hwang, K.S., Park, J., Ahn, S., Kim, T.S., 2004. Label free novel electrical detection using micromachined PZT monolithic thin film cantilever for the detection of C-reactive protein. Biosens. Bioelectron. 20, 269-75. doi:10.1016/j.bios.2004.01.024

Li, J., Shah, C.M., Withayachumnankul, W., Ung, B.S.-Y., Mitchell, A., Sriram, S., Bhaskaran, M., Chang, S., Abbott, D., 2013. Flexible terahertz metamaterials for dual-axis strain sensing. Opt. Lett. 38, 2104-6. doi:10.1364/OL.38.002104

Liu, R., Degiron, A., Mock, J.J., Smith, D.R., 2007. Negative index material composed of electric and magnetic resonators. Appl. Phys. Lett. $90,263504$. doi: $10.1063 / 1.2752120$

Liu, Y., Zhang, X., 2011. Metamaterials: a new frontier of science and technology. Chem. Soc. Rev. 40, 2494-507. doi:10.1039/c0cs00184h

Liu, Z., Xia, X., Sun, Y., Yang, H., Chen, R., Liu, B., Quan, B., Li, J., Gu, C., 2012. Visible transmission response of nanoscale complementary metamaterials for sensing applications. Nanotechnology 23, 275503-275503. doi:10.1088/0957-4484/23/27/275503

Melik, R., Unal, E., Perkgoz, N.K., Puttlitz, C., Demir, H.V., 2010. Metamaterial-based wireless RF-MEMS strain sensors, in: 2010 IEEE Sensors. IEEE, pp. 2173-2176. doi:10.1109/ICSENS.2010.5690582

Mems for Biomedical Applications, 2012. . Elsevier Science.

Metamaterials: Physics and Engineering Explorations, 2006. . John Wiley \& Sons.

Muhammad-Tahir, Z., Alocilja, E.C., 2004. A Disposable Biosensor for Pathogen Detection in Fresh Produce Samples. Biosyst. Eng. 88, 145-151. doi:10.1016/j.biosystemseng.2004.03.005

Murphy, L., 2006. Biosensors and bioelectrochemistry. Curr. Opin. Chem. Biol. 10, 177-84. doi:10.1016/j.cbpa.2006.02.023

Mutlu, M., Akosman, A.E., Serebryannikov, A.E., Ozbay, E., 2011. Asymmetric chiral metamaterial circular polarizer based on four U-shaped split ring resonators. Opt. Lett. 36, 1653. doi:10.1364/OL.36.001653

Nagel, M., Haring Bolivar, P., Brucherseifer, M., Kurz, H., Bosserhoff, A., Büttner, R., 2002. Integrated planar terahertz resonators for femtomolar sensitivity label-free detection of DNA hybridization. Appl. Opt. 41, 2074. doi:10.1364/AO.41.002074

Nanostructured Materials and Coatings for Biomedical and Sensor Applications, 2012. . Springer Science \& Business Media.

Naqui, J., Durán-Sindreu, M., Martín, F., 2011. Novel sensors based on the symmetry properties of split ring resonators (SRRs). Sensors (Basel). 11, 7545-53. doi:10.3390/s110807545

O’Hara, J.F., Singh, R., Brener, I., Smirnova, E., Han, J., Taylor, A.J., Zhang, W., 2008. Thin-film sensing with planar terahertz metamaterials: sensitivity and limitations. Opt. Express 16, 1786. doi:10.1364/OE.16.001786

Oh, B.-K., Lee, W., Kim, Y.-K., Lee, W.H., Choi, J.-W., 2004. Surface plasmon resonance immunosensor using self-assembled protein G for the detection of Salmonella paratyphi. J. Biotechnol. 111, 1-8. doi:10.1016/j.jbiotec.2004.02.010

Padilla, W.J., Basov, D.N., Smith, D.R., 2006. Negative refractive index metamaterials. Mater. Today 9, 28-35. doi:10.1016/S1369-7021(06)71573-5

Pendry, J., 2006. Photonics: metamaterials in the sunshine. Nat. Mater. 5, 599-600. doi:10.1038/nmat 1697

Pendry, J.B., 2000. Negative Refraction Makes a Perfect Lens. Phys. Rev. Lett. 85, 3966-3969. doi:10.1103/PhysRevLett.85.3966

Pendry, J.B., Holden, A.J., Robbins, D.J., Stewart, W.J., 1999. Magnetism from conductors and enhanced nonlinear phenomena. IEEE Trans. Microw. Theory Tech. 47, 2075-2084. doi:10.1109/22.798002

Pendry, J.B., Schurig, D., Smith, D.R., 2006. Controlling electromagnetic fields. Science 312, 1780-2. doi:10.1126/science.1125907

Pickup, J.C., Hussain, F., Evans, N.D., Rolinski, O.J., Birch, D.J.S., 2005. Fluorescence-based glucose sensors. Biosens. Bioelectron. 20 , $2555-65$. doi:10.1016/j.bios.2004.10.002

Pitchappa, P., Manjappa, M., Ho, C.P., Qian, Y., Singh, R., Singh, N., Lee, C., 2016. Active control of near-field coupling in conductively coupled microelectromechanical system metamaterial devices. Appl. Phys. Lett. 108, 111102. doi:10.1063/1.4943974

Puentes, M., Weiss, C., Schussler, M., Jakoby, R., 2011. Sensor array based on split ring resonators for analysis of organic tissues, in: 2011 IEEE MTT-S International Microwave Symposium. IEEE, pp. 1-4. doi:10.1109/MWSYM.2011.5972633

Ramakrishna, S.A., 2005. Physics of negative refractive index materials. Reports Prog. Phys. 68, 449-521. doi:10.1088/0034-4885/68/2/R06

Rawat, V., Dhobale, S., Kale, S.N., 2014. Ultra-fast selective sensing of ethanol and petrol using microwave-range metamaterial complementary splitring resonators. J. Appl. Phys. 116, 164106. doi:10.1063/1.4900438

Rawat, V., Kitture, R., Kumari, D., Rajesh, H., Banerjee, S., Kale, S.N., 2016. Hazardous materials sensing: An electrical metamaterial approach. J. 
Magn. Magn. Mater. 415, 77-81.doi:10.1016/j.jmmm.2015.11.023

Rawat, V., Nadkarni, V., Kale, S.N., Hingane, S., Wani, S., Rajguru, C., 2015. Calibration and optimization of a metamaterial sensor for hybrid fuel detection, in: 2015 2nd International Symposium on Physics and Technology of Sensors (ISPTS). IEEE, pp. 257-259. doi:10.1109/ISPTS.2015.7220124

Rebello, K.J., 2004. Applications of MEMS in Surgery. Proc. IEEE 92, 43-55. doi:10.1109/JPROC.2003.820536

Sang-Gil Park, Ki-Hun Jeong, 2014. High performance label-free biosensing by all dielectric metamaterial, in: 2014 International Conference on Optical MEMS and Nanophotonics. IEEE, pp. 193-194. doi:10.1109/OMN.2014.6924590

Schaefer, M., Nowak, K., Kherad, B., Gross, W., Post, S., Gebhard, M.M., 2004. Monitoring water content of rat lung tissuein vivo using microwave reflectometry. Med. Biol. Eng. Comput. 42, 577-580. doi:10.1007/BF02347537

Schueler, M., Mandel, C., Puentes, M., Jakoby, R., 2012a. Metamaterial Inspired Microwave Sensors. IEEE Microw. Mag. 13, 57-68. doi:10.1109/MMM.2011.2181448

Schueler, M., Mandel, C., Puentes, M., Jakoby, R., 2012b. Metamaterial Inspired Microwave Sensors. IEEE Microw. Mag. 13, 57-68. doi:10.1109/MMM.2011.2181448

Schussler, M., Mandel, C., Puentes, M., Jakoby, R., 2011. Capacitive level monitoring of layered fillings in vessels using composite right/left-handed transmission lines, in: 2011 IEEE MTT-S International Microwave Symposium. IEEE, pp. 1-4. doi:10.1109/MWSYM.2011.5972632

Shelby, R.A., Smith, D.R., Schultz, S., 2001. Experimental verification of a negative index of refraction. Science 292, 77-9. doi:10.1126/science.1058847

Singh, A., Kaushik, A., Kumar, R., Nair, M., Bhansali, S., 2014. Electrochemical sensing of cortisol: a recent update. Appl. Biochem. Biotechnol. 174, 1115-26. doi:10.1007/s12010-014-0894-2

Singh, A., Pasha, S.K., Manickam, P., Bhansali, S., 2016. Single-domain antibody based thermally stable electrochemical immunosensor. Biosens. Bioelectron. 83, 162-168. doi:10.1016/j.bios.2016.04.054

Singh, R., Al-Naib, I.A.I., Koch, M., Zhang, W., 2010. Asymmetric planar terahertz metamaterials. Opt. Express 18, 13044-50. doi:10.1364/OE.18.013044

Smith, D.R., Kroll, N., 2000. Negative refractive index in left-handed materials. Phys. Rev. Lett. 85, 2933-6. doi:10.1103/PhysRevLett.85.2933

Smith, D.R., Pendry, J.B., Wiltshire, M.C.K., 2004. Metamaterials and negative refractive index. Science 305, 788-92. doi:10.1126/science.1096796

Spada, L. La, Iovine, R., Vegni, L., 2012. Metamaterial-based sensor for hemoglobin measurements, in: 2012 Loughborough Antennas \& Propagation Conference (LAPC). IEEE, pp. 1-4. doi:10.1109/LAPC.2012.6403007

Sun, J., Huang, M., Yang, J.-J., Li, T.-H., Lan, Y.-Z., 2011. A microring resonator based negative permeability metamaterial sensor. Sensors (Basel). 11, 8060-71. doi:10.3390/s110808060

Tao, H., Strikwerda, A.C., Liu, M., Mondia, J.P., Ekmekci, E., Fan, K., Kaplan, D.L., Padilla, W.J., Zhang, X., Averitt, R.D., Omenetto, F.G., 2010. Performance enhancement of terahertz metamaterials on ultrathin substrates for sensing applications. Appl. Phys. Lett. 97, 261909. doi:10.1063/1.3533367

Tao, Y., Wang, G., 2012. Conformal hyperthermia of superficial tumor with left-handed metamaterial lens applicator. IEEE Trans. Biomed. Eng. 59, 3525-30. doi:10.1109/TBME.2012.2218108

Torun, H., Cagri Top, F., Dundar, G., Yalcinkaya, A.D., 2014. An antenna-coupled split-ring resonator for biosensing. J. Appl. Phys. 116, 124701. doi:10.1063/1.4896261

Vabbina, P.K., Kaushik, A., Pokhrel, N., Bhansali, S., Pala, N., 2015. Electrochemical cortisol immunosensors based on sonochemically synthesized zinc oxide 1D nanorods and 2D nanoflakes. Biosens. Bioelectron. 63, 124-130. doi:10.1016/j.bios.2014.07.026

Velazquez-Ahumada, M.C., Freire, M.J., Marques, R., 2011. Metamaterial applicator for microwave hyperthermia, in: 2011 XXXth URSI General Assembly and Scientific Symposium. IEEE, pp. 1-4. doi:10.1109/URSIGASS.2011.6050641

Veselago, V.G., 1968. THE ELECTRODYNAMICS OF SUBSTANCES WITH SIMULTANEOUSLY NEGATIVE VALUES OF \$lepsilon\$ AND $\mu$. Sov. Phys. Uspekhi 10, 509-514. doi:10.1070/PU1968v010n04ABEH003699

Viveros, L., Paliwal, S., McCrae, D., Wild, J., Simonian, A., 2006. A fluorescence-based biosensor for the detection of organophosphate pesticides and chemical warfare agents. Sensors Actuators B Chem. 115, 150-157. doi:10.1016/j.snb.2005.08.032

Vrba, D., Rodrigues, D.B., Vrba (Jr.), J., Stauffer, P.R., 2016. Metamaterial Antenna Arrays for Improved Uniformity of Microwave Hyperthermia Treatments. Prog. Electromagn. Res. 156, 1-12.

Wang, G., Fang, J., Dong, X., 2007. Resolution of Near-Field Microwave Target Detection and Imaging by Using Flat LHM Lens. IEEE Trans. Antennas Propag. 55, 3534-3541. doi:10.1109/TAP.2007.910365

Wang, J., 2006. Electrochemical biosensors: towards point-of-care cancer diagnostics. Biosens. Bioelectron. 21, 1887-92. doi:10.1016/j.bios.2005.10.027

Wang, S., Xia, L., Mao, H., Jiang, X., Yan, S., Wang, H., Wei, D., Cui, H., Du, C., 2016. Terahertz biosensing based on a polarization-insensitive metamaterial. IEEE Photonics Technol. Lett. 28, 1-1. doi:10.1109/LPT.2016.2522473

Wellenzohn, M., Brandl, M., 2015. A Theoretical Design of a Biosensor Device Based on Split Ring Resonators for Operation in the Microwave Regime. Procedia Eng. 120, 865-869. doi:10.1016/j.proeng.2015.08.737

Winter, W., Höhne, G.W.., 2003. Chip-calorimeter for small samples. Thermochim. Acta 403, 43-53. doi:10.1016/S0040-6031(03)00159-X

Withayachumnankul, W., Jaruwongrungsee, K., Tuantranont, A., Fumeaux, C., Abbott, D., 2013. Metamaterial-based microfluidic sensor for dielectric characterization. Sensors Actuators A Phys. 189, 233-237. doi:10.1016/j.sna.2012.10.027

Wu, X., Quan, B., Pan, X., Xu, X., Lu, X., Gu, C., Wang, L., 2013. Alkanethiol-functionalized terahertz metamaterial as label-free, highly-sensitive and specific biosensor. Biosens. Bioelectron. 42, 626-31. doi:10.1016/j.bios.2012.10.095

Wuttig, M., Yamada, N., 2007. Phase-change materials for rewriteable data storage. Nat. Mater. 6, 824-832. doi:10.1038/nmat2009

Xu, X., Peng, B., Li, D., Zhang, J., Wong, L.M., Zhang, Q., Wang, S., Xiong, Q., 2011. Flexible Visible-Infrared Metamaterials and Their Applications in Highly Sensitive Chemical and Biological Sensing.

Yang, M.H. and J., 2011. Wave Propagation. InTech. doi:10.5772/584

Yang, Y., Huang, R., Cong, L., Zhu, Z., Gu, J., Tian, Z., Singh, R., Zhang, S., Han, J., Zhang, W., 2011. Modulating the fundamental inductivecapacitive resonance in asymmetric double-split ring terahertz metamaterials. Appl. Phys. Lett. 98, 121114. doi:10.1063/1.3571288

Yang, Z., Zhou, Q., Long, W., Li, C., Shi, Y., Wu, A., Liu, J., Zhang, C., 2016. Study of asymmetric U-shaped resonator at terahertz frequencies. Opt. Commun. 368, 119-122. doi:10.1016/j.optcom.2016.01.087

Yoshida, H., Ogawa, Y., Kawai, Y., Hayashi, S., Hayashi, A., Otani, C., Kato, E., Miyamaru, F., Kawase, K., 2007. Terahertz sensing method for protein detection using a thin metallic mesh. Appl. Phys. Lett. 91, 253901. doi:10.1063/1.2825411

Yoshida, S., Suizu, K., Kato, E., Nakagomi, Y., Ogawa, Y., Kawase, K., 2009. A high-sensitivity terahertz sensing method using a metallic mesh with unique transmission properties. J. Mol. Spectrosc. 256, 146-151. doi:10.1016/j.jms.2009.04.009

Zarifi, M.H., Farsinezhad, S., Abdolrazzaghi, M., Daneshmand, M., Shankar, K., 2016. Selective microwave sensors exploiting the interaction of analytes with trap states in $\mathrm{TiO}_{2}$ nanotube arrays. Nanoscale 8, 7466-7473. doi:10.1039/C5NR06567D 
Zhang, J., Grischkowsky, D., 2004. Waveguide terahertz time-domain spectroscopy of nanometer water layers. Opt. Lett. 29, 1617. doi:10.1364/OL.29.001617

Ziegler, C., 2004. Nanotechnologies for the biosciences. Anal. Bioanal. Chem. 379. doi:10.1007/s00216-004-2709-8

\section{Graphical Abstract}

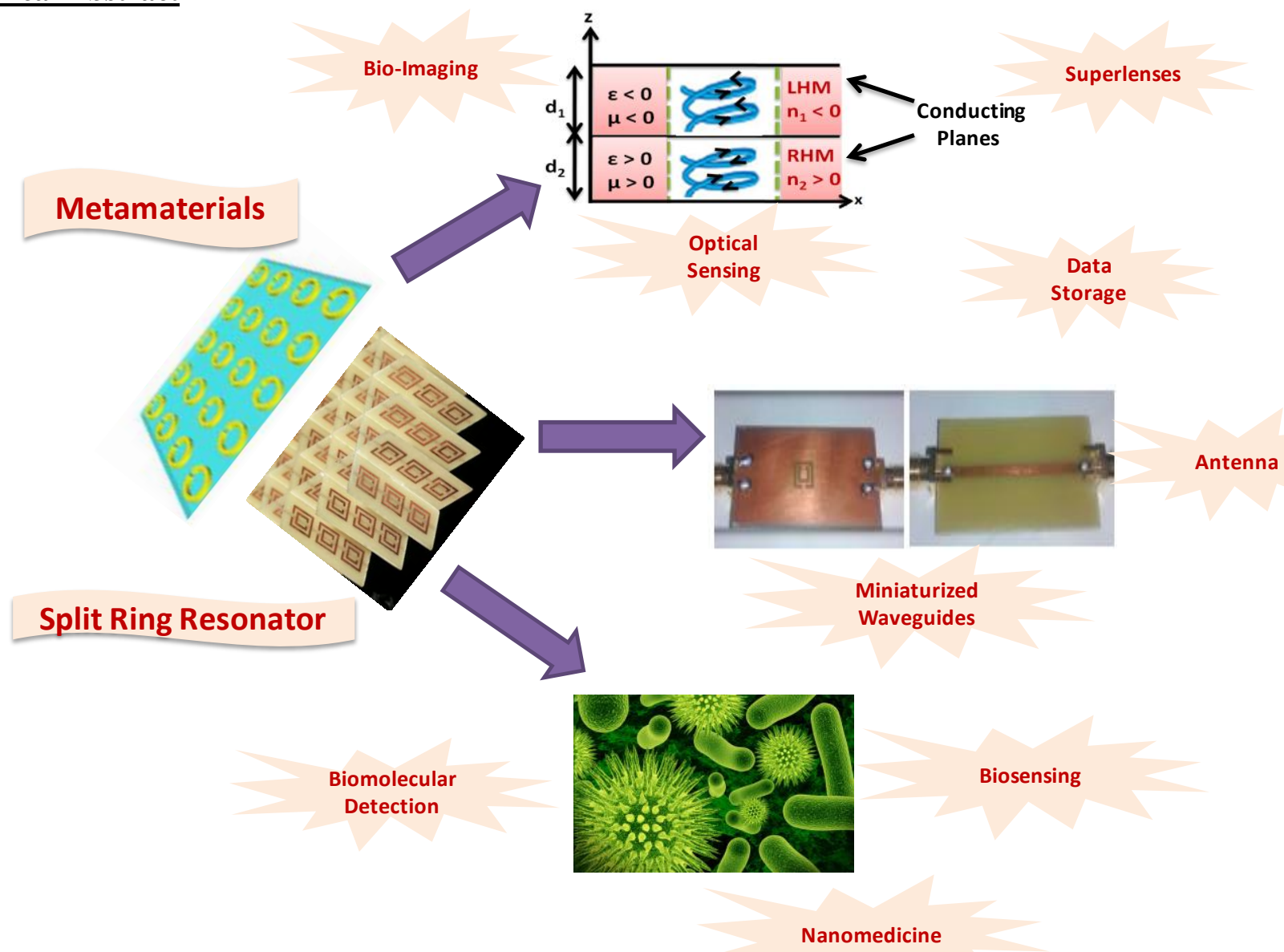




\section{$\underline{\text { Graphical Abstract }}$}

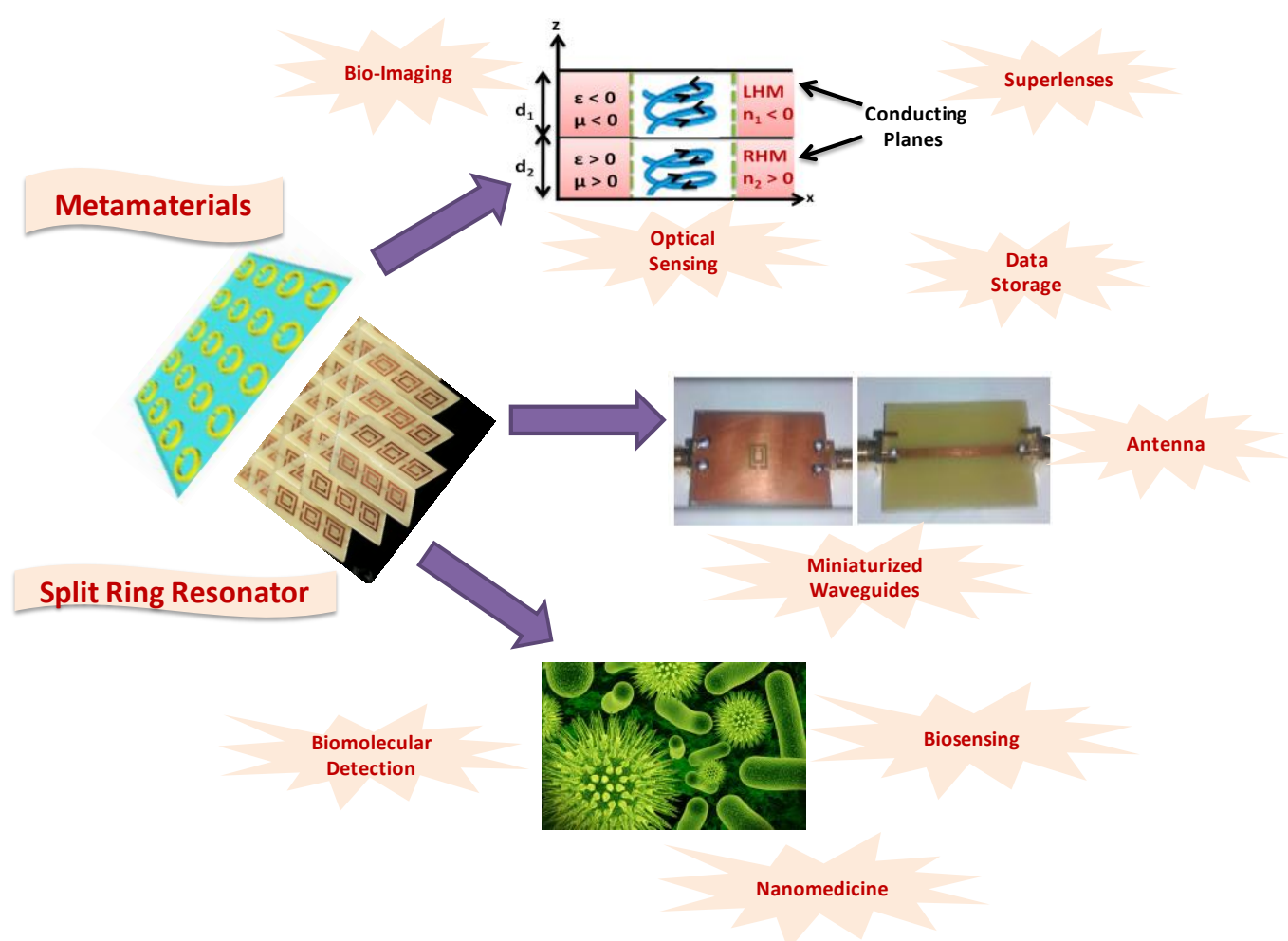

\section{Figures}

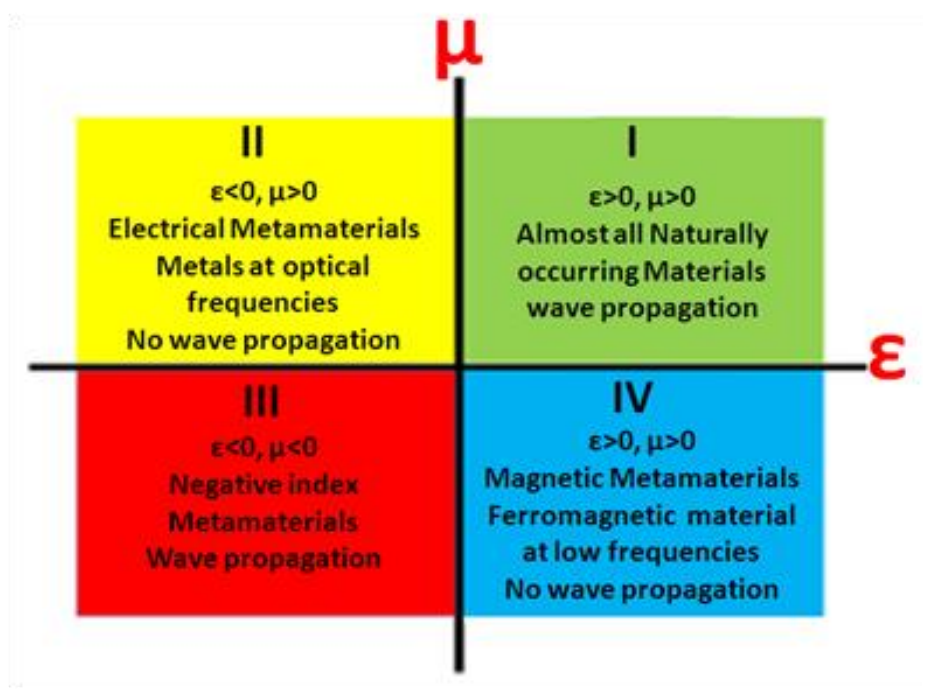

Figure 1. Graph of $\varepsilon$ vs. $\mu$, showing various quadrants and the materials' behaviour in each of these divisions the graph of behaviour in each of these divisions 


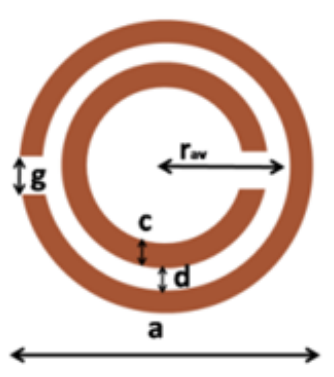

(a)

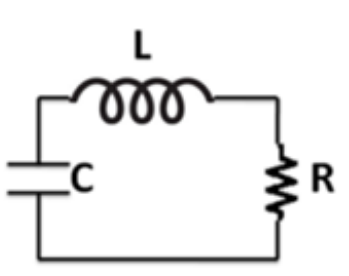

(b)

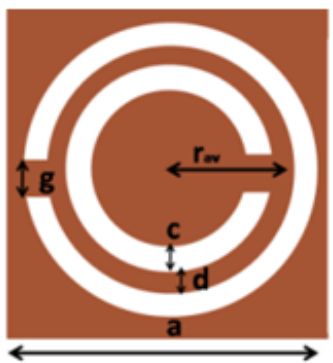

(c)

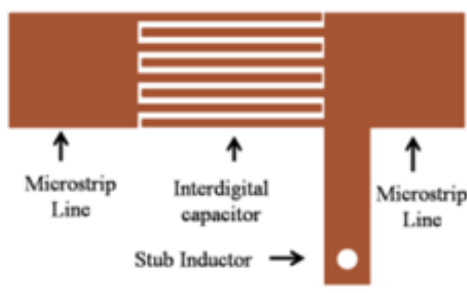

(d)

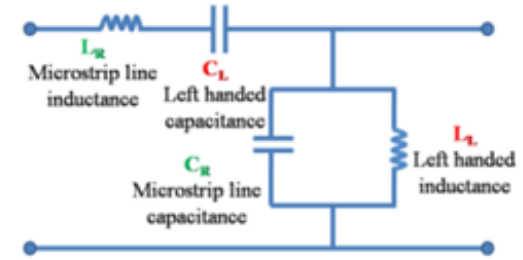

(e)

Figure 2. (a) Schematic of a double-turn split-ring resonator with its dimension (b) equivalent RLC tank circuit of a single split ring resonator, valid when it is electrically small (c) Schematic of a double complementary split-ring resonator and (d) Layout of the unit cell of the microstrip Left Handed-Transmission Line prototype, including a series interdigital capacitor and a shunt shorted stub inductor for left handedness (e) Equivalent circuit of Composite Right Left Handed(CRLH) metamaterial transmission line

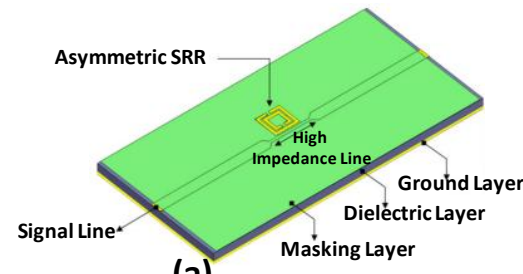

(a)
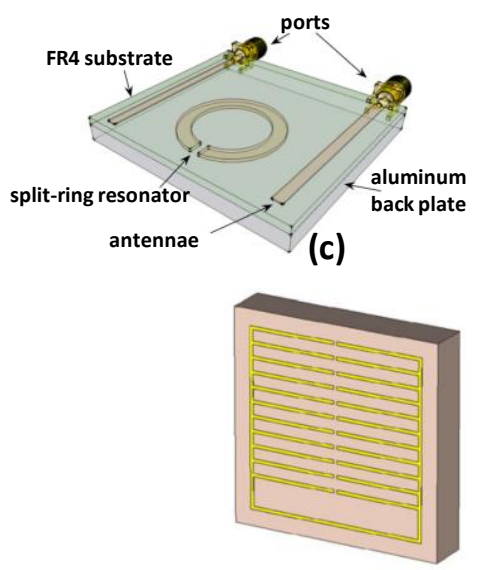

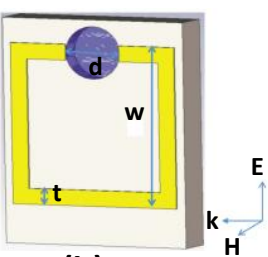

(b)

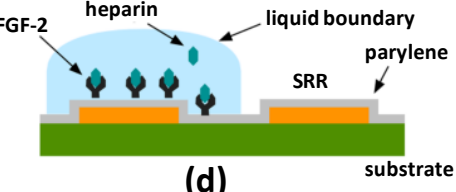

(d)

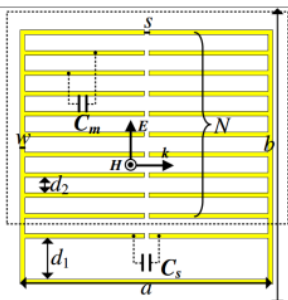

(e)

Figure 3. (a) Schematic of a SRR based on a high impedance microstrip line for label free detection (b) Schematic of the SRR structure printed on a circuit board for bioassay application. (c) Biosensor structure and integrated monopole antennas on a dielectric substrate (d) experimental setup presenting the functionalization of the device with bio-molecules (e) Nested Split Ring 


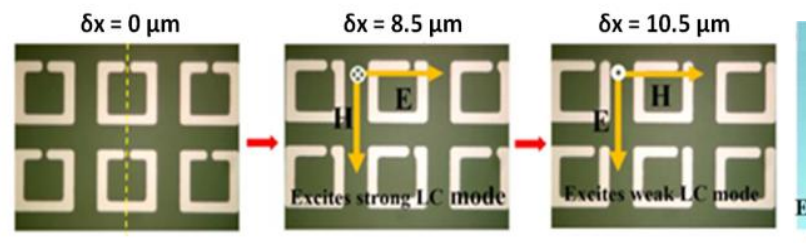

(a)

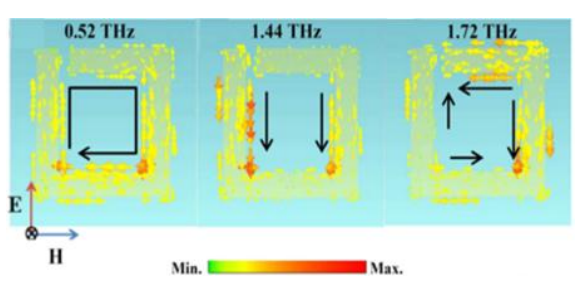

(b)
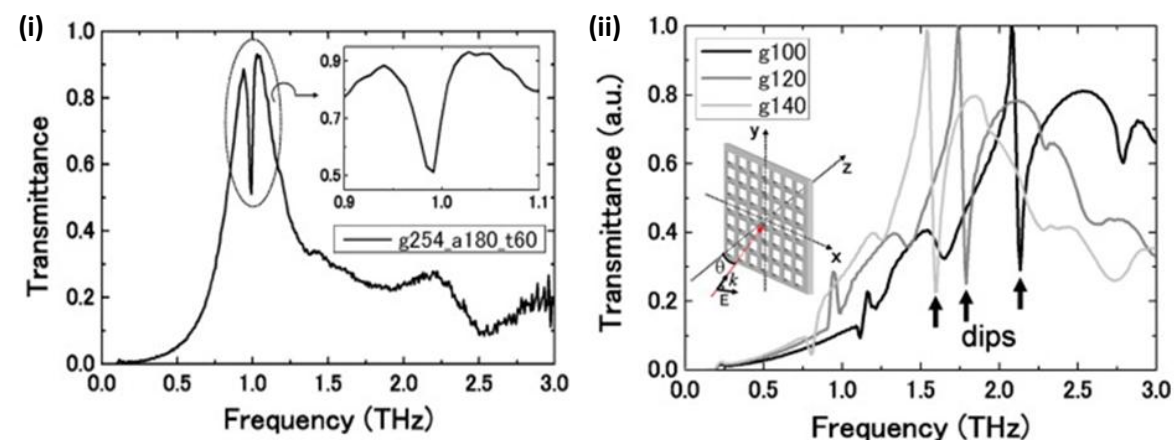

(c)

Figure 4. (a) Microscopic image of SRR arrays with increase in gap in the SRR by different values of ' $\delta$ ' from 0 um to 10.5 um from the center, (b) Surface current distribution for asymmetry parameter $\delta \mathrm{x}=10.5 \mu \mathrm{m}$ for vertical E field polarization at different frequencies of weak LC resonance, dipole resonance and quadrupole resonance, (c) [i] Transmission spectra of metallic mesh [ii] Dip frequency reliance on the opening ratio for the square-lattice metallic mesh
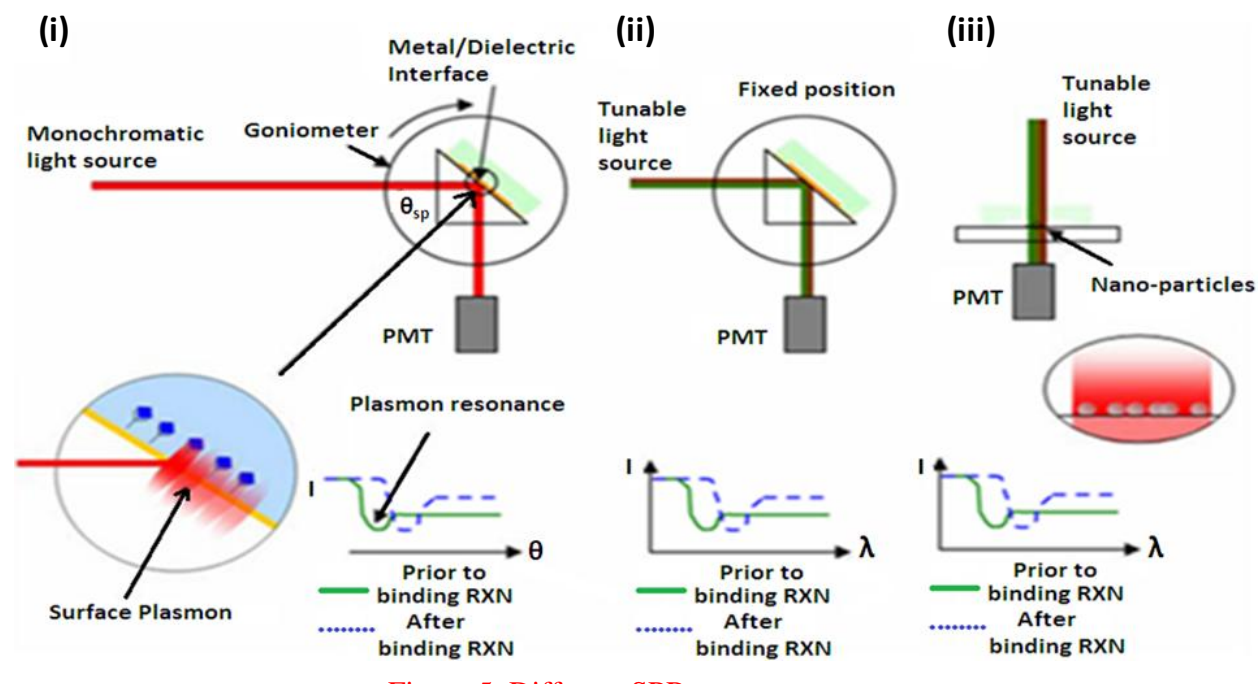

Figure 5. Different SPR sensor systems 


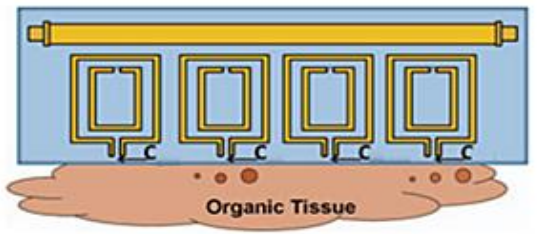

(a)

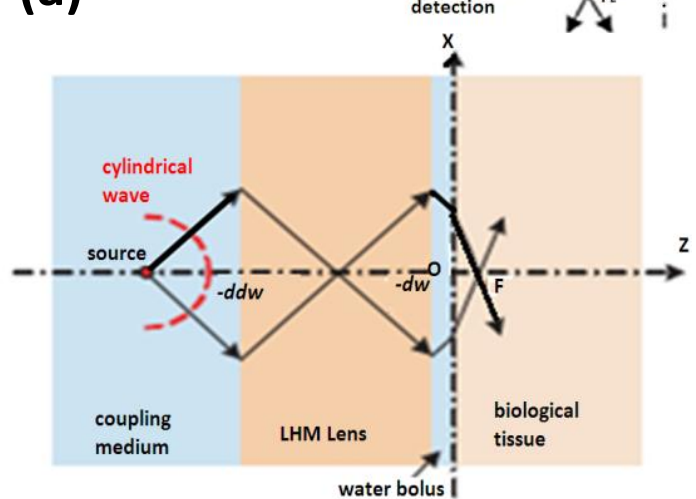

(c)

Figure 6. (a) SRR sensor array for analysis of organic tissues (b) Design for target detection and imaging with flat LHM lens at microwave frequencies (c) Hyperthermia scheme with flat LHM lens

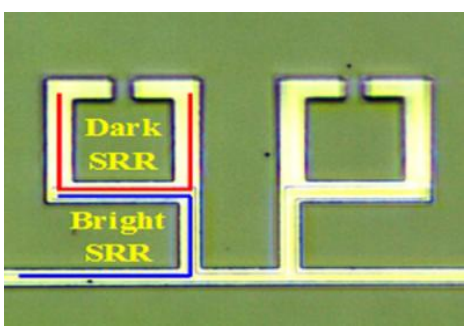

(a)

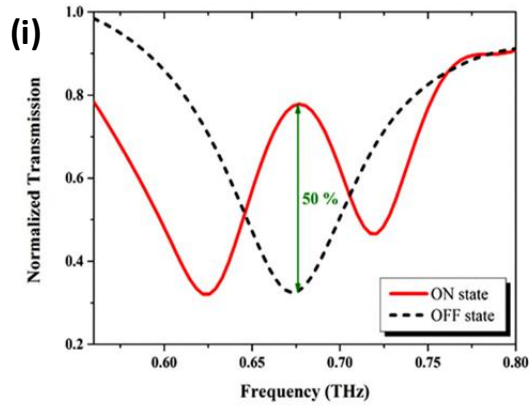

(i)

(ii)
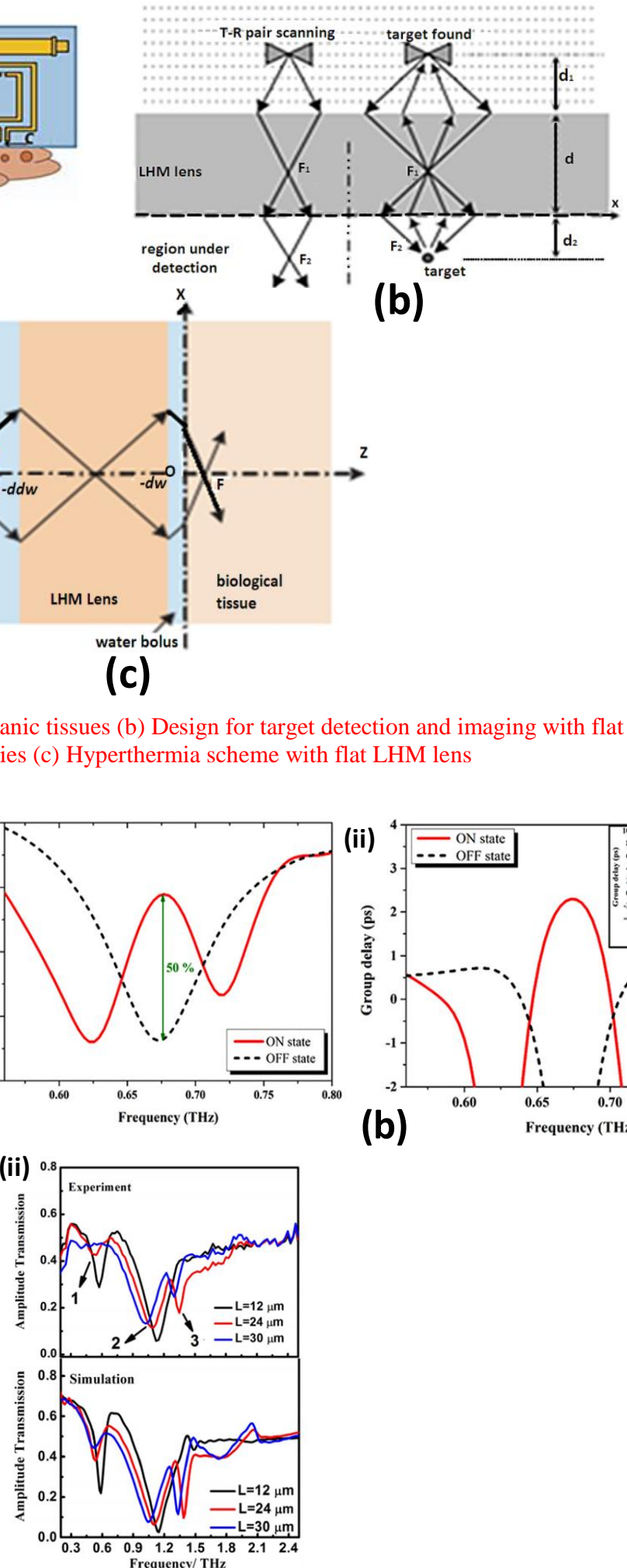

(c)

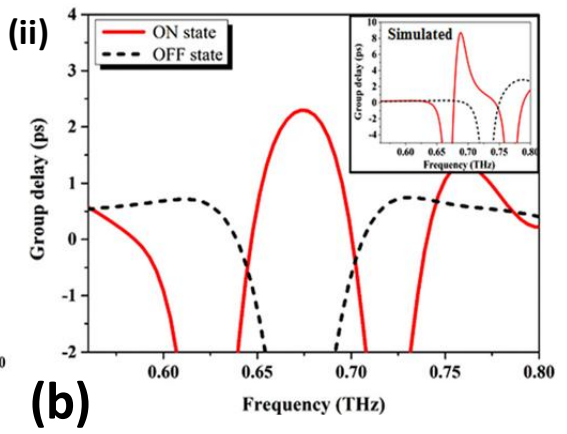

(b)

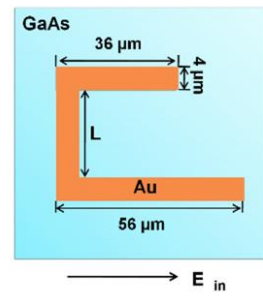

Fig 7: (a) Optical microscopic image of conductively coupled MEMS device with curved cantilevers (b) Measured (i) THz response (ii) Group delay of conductively coupled MEMS device (c) (i) Schematic of asymmetric design structure with perpendicular incident polarization (ii) Obtained amplitude transmission spectra 